Pacific

Journal of

Mathematics

\title{
KLEIN FOUR-SUBGROUPS OF \\ LIE ALGEBRA AUTOMORPHISMS
}

JING-SONG HUANG AND JUN YU 


\title{
KLEIN FOUR-SUBGROUPS OF LIE ALGEBRA AUTOMORPHISMS
}

\author{
JING-SONG HUANG AND JUN YU
}

\begin{abstract}
We classify the Klein four-subgroups $\Gamma$ of $\operatorname{Aut}\left(\mathfrak{u}_{0}\right)$ for each compact simple Lie algebra $\mathfrak{u}_{0}$ up to conjugation, by calculating the symmetric subgroups $\operatorname{Aut}\left(\mathfrak{u}_{0}\right)^{\theta}$ and their involution classes. This leads to a new approach to the classification of semisimple symmetric pairs and $\mathbb{Z}_{2} \times \mathbb{Z}_{2}$-symmetric spaces. We also determine the fixed point subgroups $\operatorname{Aut}\left(\mathfrak{u}_{0}\right)^{\Gamma}$.
\end{abstract}

\section{Introduction}

Riemannian symmetric pairs were classified by Élie Cartan (see [Carter 1993], for example) and the more general semisimple symmetric pairs were classified by Marcel Berger [1957]. The algebraic structure of semisimple symmetric spaces is even more interesting for geometric and analytic reasons. Some of the recent works are Ōshima and Sekiguchi's classification [1984] of reduced root systems and Helminck's classification [1988] for algebraic groups. Most recently some new approaches to the classification and the parametrization of semisimple symmetric pairs were given in [Huang 2002] by using admissible quadruplets and in [Chuah and Huang 2010] by using double Vogan diagrams.

In this paper we study semisimple symmetric spaces from a different point of view - by determining the Klein four-subgroups in Lie algebra automorphisms. Let $\mathfrak{u}_{0}$ be a compact simple Lie algebra and $\mathfrak{g}$ be its complexification. Denote by $\operatorname{Aut}\left(\mathfrak{u}_{0}\right)$ the automorphism group of $\mathfrak{u}_{0}$. For any involution $\theta$ in $\operatorname{Aut}\left(\mathfrak{u}_{0}\right)$, we first determine the centralizer $\operatorname{Aut}\left(\mathfrak{u}_{0}\right)^{\theta}$ of $\theta$, which is a symmetric subgroup. By understanding the conjugacy classes of involutions in $\operatorname{Aut}\left(\mathfrak{u}_{0}\right)^{\theta}$, we proceed to classify Klein four-subgroups $\Gamma$ of $\operatorname{Aut}\left(\mathfrak{u}_{0}\right)$ up to conjugation. This gives a new approach to the classification of commuting pairs of involutive automorphisms of $\mathfrak{u}_{0}$ or $\mathfrak{g}$. We note that the ordered commuting pairs of involutions correspond to

The research work described in this paper was partially supported by a Research Grant from Research Grant Council of HKSAR, China; the second author's current work is supported by a grant from SNF (Schweizerischer Nationalfonds). Finally, we would like to thank the anonymous referee for his/her careful reading and helpful comments.

MSC2010: primary 20E45; secondary 53C35.

Keywords: automorphism group, involution, symmetric subgroup, Klein four-group, involution type. 
Berger's classification of semisimple symmetric pairs.

If $\Gamma$ is a finite abelian subgroup of the automorphism group of a Lie group $G$, then the homogeneous space $G / H$ is called a $\Gamma$-symmetric space provided that $\left(G^{\Gamma}\right)_{0} \subseteq H \subseteq G^{\Gamma}$; see [Lutz 1981]. In the case of $\Gamma=\mathbb{Z}_{2}$ this is a symmetric space and in the case of $\Gamma=\mathbb{Z}_{k}$ it is the $k$-symmetric space studied in [Wolf and Gray 1968]. In the case of $\Gamma=\mathbb{Z}_{2} \times \mathbb{Z}_{2}$ it is the Klein four-group; $\mathbb{Z}_{2} \times \mathbb{Z}_{2}$-symmetric spaces were studied in [Bahturin and Goze 2008; Kollross 2009]. This paper contains a complete list of all $\mathbb{Z}_{2} \times \mathbb{Z}_{2}$-symmetric pairs and our method is very different from theirs. Finally, we determine the fixed point subgroups $\operatorname{Aut}\left(\mathfrak{u}_{0}\right)^{\Gamma}$.

\section{Preliminaries}

2A. Complex semisimple Lie algebras and Dynkin diagrams. Let $\mathfrak{g}$ be a complex semisimple Lie algebra and $\mathfrak{h}$ a Cartan subalgebra. Then $\mathfrak{g}$ has a root-space decomposition

$$
\mathfrak{g}=\mathfrak{h} \oplus\left(\bigoplus_{\alpha \in \Delta} \mathfrak{g}_{\alpha}\right),
$$

where $\Delta=\Delta(\mathfrak{g}, \mathfrak{h})$ is the root system of $\mathfrak{g}$ and $\mathfrak{g}_{\alpha}$ is the root space of the root $\alpha \in \Delta$. Let $B$ be the Killing form on $\mathfrak{g}$. It is a nondegenerate symmetric form. The restriction of $B$ to $\mathfrak{h}$ is also nondegenerate. For any $\lambda \in \mathfrak{h}^{*}$, let $H_{\lambda} \in \mathfrak{h}$ be determined by

$$
B\left(H_{\lambda}, H\right)=\lambda(H) \text { for all } H \in \mathfrak{h} .
$$

For any $\lambda, \mu \in \mathfrak{h}^{*}$, define $\langle\lambda, \mu\rangle:=B\left(H_{\lambda}, H_{\mu}\right)$.

For any root $\alpha$, we have

$$
H_{\alpha} \in \mathfrak{h}
$$

Define

$$
H_{\alpha}^{\prime}=\frac{2}{\alpha\left(H_{\alpha}\right)} H_{\alpha}
$$

which is called a coroot; let

$$
0 \neq X_{\alpha} \in \mathfrak{g}_{\alpha}
$$

be any nonzero vector (recall that $\operatorname{dim} \mathfrak{g}_{\alpha}=1$ ), which is called a root vector of the root $\alpha$. The notation $H_{\alpha}, H_{\alpha}^{\prime}, X_{\alpha}$ will be used frequently in this paper.

Note that, for any $\alpha, \beta \in \Delta$,

$$
\begin{gathered}
\langle\alpha, \beta\rangle=B\left(H_{\alpha}, H_{\beta}\right)=\beta\left(H_{\alpha}\right)=\alpha\left(H_{\beta}\right) \in \mathbb{R}, \\
\langle\alpha, \alpha\rangle=B\left(H_{\alpha}, H_{\alpha}\right)=\alpha\left(H_{\alpha}\right) \neq 0,
\end{gathered}
$$

and $2\langle\alpha, \beta\rangle /\langle\beta, \beta\rangle \in \mathbb{Z}$. We also note that $\operatorname{span}_{\mathbb{R}}\{\alpha \mid \alpha \in \Delta\} \subset \mathfrak{h}^{*}$ is a real vector space of dimension equal to $r=\operatorname{rank} \mathfrak{g}=\operatorname{dim}_{\mathbb{C}} \mathfrak{h}$; see [Knapp 2002, pp. 140-162]. 
We set $A_{\alpha, \beta}=2\langle\alpha, \beta\rangle /\langle\beta, \beta\rangle=\alpha\left(H_{\beta}^{\prime}\right)$. Then

$$
\left[H_{\alpha}^{\prime}, X_{\beta}\right]=\beta\left(H_{\alpha}^{\prime}\right) X_{\beta}=\frac{2\langle\alpha, \beta\rangle}{\langle\alpha, \alpha\rangle} X_{\beta}=A_{\beta, \alpha} X_{\beta}
$$

Choose a lexicography order of $\operatorname{span}_{\mathbb{R}}\{\alpha \mid \alpha \in \Delta\}$ to get a positive system $\Delta^{+}$ and a simple system $\Pi$. Let

$$
\Pi=\left\{\alpha_{1}, \alpha_{2}, \ldots, \alpha_{r}\right\}
$$

For brevity, we write

$$
H_{i}, H_{i}^{\prime}
$$

instead of $H_{\alpha_{i}}, H_{\alpha_{i}}^{\prime}$ for a simple root $\alpha_{i}$.

Draw $A_{\alpha, \beta} A_{\beta, \alpha}$ edges to connect any two distinct simple roots $\alpha$ and $\beta$, and draw an arrow from $\alpha$ to $\beta$ if $\langle\alpha, \alpha\rangle>\langle\beta, \beta\rangle$; this gives us a graph. This graph is connected if and only if $\mathfrak{g}$ is a simple Lie algebra; in this case it is called the Dynkin diagram of $\mathfrak{g}$. In this paper, we always follow Bourbaki numbering to order the simple roots; see [Bourbaki 2002, pp. 265-300]. The following are all the possible (connected) Dynkin diagrams. ${ }^{1}$

$\mathrm{A}_{n}$
$\mathrm{~B}_{n}$
$\mathrm{C}_{n}$
$\mathrm{D}_{n}$
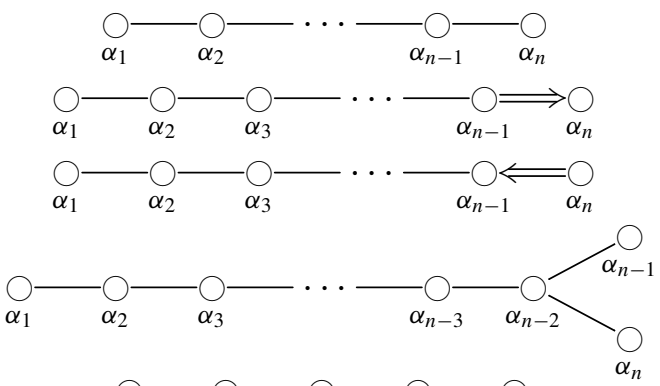

$\mathrm{E}_{6}$

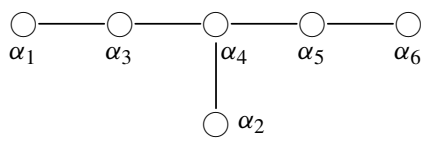

$\mathrm{E}_{7}$

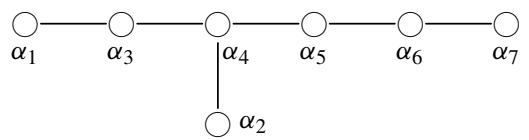

$\mathrm{E}_{8}$

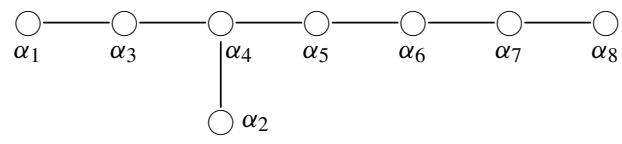

$\mathrm{F}_{4}$

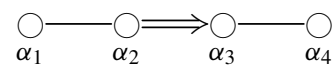

$\mathrm{G}_{2}$

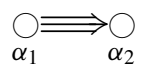

${ }^{1}$ These diagrams are drawn by using a Latex package of Professor Jiu-Kang Yu. We are grateful to him for the kind permission to use this package. 
Let $\operatorname{Aut}(\mathfrak{g})$ be the group of all complex linear automorphisms of $\mathfrak{g}$ and $\operatorname{Int}(\mathfrak{g})$ be the subgroup of inner automorphisms. We define

$$
\operatorname{Out}(\mathfrak{g}):=\operatorname{Aut}(\mathfrak{g}) / \operatorname{Int}(\mathfrak{g}) .
$$

The exponential map exp $: \mathfrak{g} \rightarrow \operatorname{Aut}(\mathfrak{g})$ is given by

$$
\exp (X)=\exp (\operatorname{ad}(X)) \text { for all } X \in \mathfrak{g}=\operatorname{Lie}(\operatorname{Aut}(\mathfrak{g})) \text {. }
$$

2B. A compact real form. One can normalize the root vectors $\left\{X_{\alpha}, X_{-\alpha}\right\}$ so that $B\left(X_{\alpha}, X_{-\alpha}\right)=2 / \alpha\left(H_{\alpha}\right)$. Then $\left[X_{\alpha}, X_{-\alpha}\right]=H_{\alpha}^{\prime}$. Moreover, one can normalize $\left\{X_{\alpha}\right\}$ appropriately, such that

$$
\mathfrak{u}_{0}=\operatorname{span}_{\mathbb{R}}\left\{X_{\alpha}-X_{-\alpha}, i\left(X_{\alpha}+X_{-\alpha}\right), i H_{\alpha}: \alpha \in \Delta^{+}\right\}
$$

is a compact real form of $\mathfrak{g}$ [Knapp 2002, pp. 348-354]. Define

$$
\theta(X+i Y):=X-i Y \text { for all } X, Y \in \mathfrak{u}_{0} .
$$

Then $\theta$ is a Cartan involution of $\mathfrak{g}$ (as a real semisimple Lie algebra) and $\mathfrak{u}_{0}=\mathfrak{g}^{\theta}$ is a maximal compact subalgebra of $\mathfrak{g}$. Any other compact real form of $\mathfrak{g}$ is conjugate to $\mathfrak{u}_{0}$. Below, whenever we discuss a compact real form of $\mathfrak{g}$, we always use this compact real form $\mathfrak{u}_{0}$ in (6).

Let Aut $\left(\mathfrak{u}_{0}\right)$ be the group of automorphisms of $\mathfrak{u}_{0}$ and $\operatorname{Int}\left(\mathfrak{u}_{0}\right)$ be the subgroup of inner automorphisms. Any automorphism of $\mathfrak{u}_{0}$ extends uniquely to a holomorphic automorphism of $\mathfrak{g}, \operatorname{so} \operatorname{Aut}\left(\mathfrak{u}_{0}\right) \subset \operatorname{Aut}(\mathfrak{g})$. Similarly, $\operatorname{Int}\left(\mathfrak{u}_{0}\right) \subset \operatorname{Int}(\mathfrak{g})$. Define

$$
\Theta(f):=\theta f \theta^{-1} \quad \text { for all } f \in \operatorname{Aut}(\mathfrak{g}) .
$$

Then it is a Cartan involution of $\operatorname{Aut}(\mathfrak{g})$ with differential $\theta$. It follows that $\operatorname{Aut}\left(\mathfrak{u}_{0}\right)=$ $\operatorname{Aut}(\mathfrak{g})^{\Theta}$ and $\operatorname{Int}\left(\mathfrak{u}_{0}\right)=\operatorname{Int}(\mathfrak{g})^{\Theta}$ are maximal compact subgroups of $\operatorname{Aut}(\mathfrak{g})$ and $\operatorname{Int}(\mathfrak{g})$, respectively. We also have

$$
\operatorname{Out}\left(\mathfrak{u}_{0}\right):=\operatorname{Aut}\left(\mathfrak{u}_{0}\right) / \operatorname{Int}\left(\mathfrak{u}_{0}\right) \cong \operatorname{Out}(\mathfrak{g}) \cong \operatorname{Aut}(\Pi),
$$

where $\operatorname{Aut}(\Pi)$ is the symmetry group of the graph $\Pi$ consisting of permutations of vertices preserving the multiples of edges and directions of arrows.

2C. Notation. We denote by $\mathfrak{e}_{6}$ the compact simple Lie algebra of type $\mathbf{E}_{6}$. Let $\mathrm{E}_{6}$ be the connected and simply connected Lie group with Lie algebra $\mathfrak{e}_{6}$. Let $\mathfrak{e}_{6}(\mathbb{C})$ and $\mathrm{E}_{6}(\mathbb{C})$ denote their complexifications. Similar notation will be used for other types.

Let $Z(G)$ and $\mathfrak{z}(\mathfrak{g})$ denote the center of a group $G$ and a Lie algebra $\mathfrak{g}$, respectively, and $G_{0}$ denote the connected component of $G$ containing identity element. For Lie groups $H \subset G$, let $Z_{G}(H)$ denote the centralizer of $H$ in $G$, and for Lie algebras $\mathfrak{h} \subset \mathfrak{g}$, let $Z_{\mathfrak{g}}(\mathfrak{h})$ denote the centralizer of $\mathfrak{h}$ in $\mathfrak{g}$. Let $N_{G}(H)$ denote the normalizer 
of $H$ in $G$. For any two elements $x, y \in G$, we write $x \sim y$ to mean $x, y$ are conjugate in $G$, that is, $y=g x g^{-1}$ for some $g \in G$ and $x \sim_{H} y$ to mean $y=g x g^{-1}$ for some $g \in H$.

In the case of $G=\mathrm{E}_{6}$ or $\mathrm{E}_{7}$, let $c$ denote a nontrivial element in $Z(G)$.

In the case of $\mathfrak{u}_{0}=\mathfrak{e}_{7}$, let

$$
H_{0}^{\prime}=\frac{H_{2}^{\prime}+H_{5}^{\prime}+H_{7}^{\prime}}{2} \in i \mathfrak{e}_{7} \subset \mathfrak{e}_{7}(\mathbb{C}) .
$$

Let $\operatorname{Pin}(n)(\operatorname{Spin}(n))$ be the Pin (Spin) group in degree $n$. Write

$$
c=e_{1} e_{2} \cdots e_{n} \in \operatorname{Pin}(n) .
$$

Then $c$ is in $\operatorname{Spin}(n)$ if and only if $n$ is even; in this case $c \in Z(\operatorname{Spin}(n))$. If $n$ is odd, then $\operatorname{Spin}(n)$ has a spinor module $M$ of dimension $2^{(n-1) / 2}$. If $n$ is even, then $\operatorname{Spin}(n)$ has two spinor modules $M_{+}, M_{-}$of dimension $2^{(n-2) / 2}$. We distinguish $M_{+}$and $M_{-}$by requiring that $c$ acts on $M_{+}$as the identity when $4 \mid n$ and as multiplication by $-i$ when $4 \mid n-2$ (and thus $c$ acts on $M_{-}$as multiplication by -1 and $i$, respectively, in the same two cases).

We define the matrices

$$
\begin{gathered}
J_{m}=\left(\begin{array}{cc}
0 & I_{m} \\
-I_{m} & 0
\end{array}\right), \quad I_{p, q}=\left(\begin{array}{cc}
-I_{p} & 0 \\
0 & I_{q}
\end{array}\right), \\
I_{p, q}^{\prime}=\left(\begin{array}{cccc}
-I_{p} & 0 & 0 & 0 \\
0 & I_{q} & 0 & 0 \\
0 & 0 & -I_{p} & 0 \\
0 & 0 & 0 & I_{q}
\end{array}\right), \quad J_{p, q}=\left(\begin{array}{cccc}
0 & I_{p} & 0 & 0 \\
-I_{p} & 0 & 0 & 0 \\
0 & 0 & 0 & I_{q} \\
0 & 0 & -I_{q} & 0
\end{array}\right), \\
K_{p}=\left(\begin{array}{cccc}
0 & 0 & 0 & I_{p} \\
0 & 0 & -I_{p} & 0 \\
0 & I_{p} & 0 & 0 \\
-I_{p} & 0 & 0 & 0
\end{array}\right) .
\end{gathered}
$$

and the groups

$$
\begin{aligned}
Z_{m} & =\left\{\lambda I_{m} \mid \lambda^{m}=1\right\}, \\
Z^{\prime} & =\left\{\left(\epsilon_{1}, \epsilon_{2}, \epsilon_{3}, \epsilon_{4}\right) \mid \epsilon_{i}= \pm 1, \epsilon_{1} \epsilon_{2} \epsilon_{3} \epsilon_{4}=1\right\}, \\
\Gamma_{p, q, r, s} & =\left\langle\left(\begin{array}{cccc}
-I_{p} & 0 & 0 & 0 \\
0 & -I_{q} & 0 & 0 \\
0 & 0 & I_{r} & 0 \\
0 & 0 & 0 & I_{s}
\end{array}\right),\left(\begin{array}{cccc}
-I_{p} & 0 & 0 & 0 \\
0 & I_{q} & 0 & 0 \\
0 & 0 & -I_{r} & 0 \\
0 & 0 & 0 & I_{s}
\end{array}\right)\right\rangle .
\end{aligned}
$$




\section{Involutions}

The classical compact simple Lie algebras are as follows. For $F=\mathbb{R}, \mathbb{C}, \mathbb{M}$, let $M_{n}(F)$ be the set of $n \times n$ matrices with entries in $F$, and

$$
\begin{aligned}
& \mathfrak{s o}(n)=\left\{X \in M_{n}(\mathbb{R}) \mid X+X^{t}=0\right\}, \\
& \mathfrak{s u}(n)=\left\{X \in M_{n}(\mathbb{C}) \mid X+X^{*}=0, \operatorname{tr} X=0\right\}, \\
& \mathfrak{s p}(n)=\left\{X \in M_{n}(\mathbb{W}) \mid X+X^{*}=0\right\} .
\end{aligned}
$$

Then $\{\mathfrak{s u}(n): n \geq 3\},\{\mathfrak{s o}(2 n+1): n \geq 1\},\{\mathfrak{s p}(n): n \geq 3\},\{\mathfrak{s o}(2 n): n \geq 4\}$ represent all isomorphism classes of compact classical simple Lie algebras.

Let $\mathfrak{u}_{0}$ be a compact simple Lie algebra and $\mathfrak{g}=\left(\mathfrak{u}_{0}\right) \otimes_{\mathbb{R}} \mathbb{C}$ be its complexification. Note that the conjugacy classes of involutions in $\operatorname{Aut}\left(\mathfrak{u}_{0}\right)$ are in one-to-one correspondence with isomorphism classes of noncompact real forms of $\mathfrak{g}$, and are also in one-to-one correspondence with isomorphism classes of irreducible Riemannian symmetric pairs $\left(\mathfrak{u}_{0}, \mathfrak{k}_{0}\right)$ of compact type or $\left(\mathfrak{g}_{0}, \mathfrak{k}_{0}\right)$ of noncompact type; see [Huang 2002; Helminck 1988] and references therein. One direction of this correspondence is as follows: let $\theta$ be an involutive automorphism of a compact real simple Lie algebra $\mathfrak{u}_{0}$, and extend it to a holomorphic automorphism of $\mathfrak{g}$. Let $\mathfrak{k}_{0} \subset \mathfrak{u}_{0}$ and $i \mathfrak{p}_{0} \subset \mathfrak{u}_{0}$ (so $\mathfrak{p}_{0} \subset i \mathfrak{u}_{0}$ ) be the $+1,-1$ eigenspaces of $\theta$ on $\mathfrak{u}_{0}$, respectively. Let

$$
\mathfrak{g}_{0}=\mathfrak{k}_{0} \oplus \mathfrak{p}_{0}
$$

(this is also the Cartan decomposition of $\mathfrak{g}_{0}$ ). Then $\mathfrak{g}_{0}$ is a real simple Lie algebra (that is, a real form of $\mathfrak{g}$ ), $\left(\mathfrak{u}_{0}, \mathfrak{k}_{0}\right)$ is a Riemannian symmetric pair of compact type and $\left(\mathfrak{g}_{0}, \mathfrak{k}_{0}\right)$ is a Riemannian symmetric pair of noncompact type. The other direction of this correspondence needs a sophisticated argument.

These objects were classified by Élie Cartan in 1926. We list this classification here. Our presentation below is mainly from [Knapp 2002, pp. 408-426; Helgason 2001, pp. 515-518]. In each case, we also define a specific involution in each conjugacy class of involutions in $\operatorname{Aut}\left(\mathfrak{u}_{0}\right)$, which corresponds to a real simple Lie algebra or symmetric space. In the exceptional simple Lie algebras case, these involutions are labeled as $\sigma_{1}, \sigma_{2}, \sigma_{3}, \sigma_{4}, \sigma$ and $\tau=\sigma_{3}$ (this is used only in the $\mathrm{E}_{6}$ case). We will use this notation for involutions frequently in the rest of this paper.

The notation AI-G is Cartan notation and the notation $\mathfrak{e}_{6,-2}$, etc., is Helgason notation (with a little difference). For a real simple Lie algebra $\mathfrak{g}_{0}$ with a Cartan decomposition $\mathfrak{g}_{0}=\mathfrak{k}_{0} \oplus \mathfrak{p}_{0}$ and whose complexified Lie algebra $\mathfrak{g}$ is an exceptional simple Lie algebra, Helgason [2001, pp. 517-518] made an interesting observation: the isomorphism type of $\mathfrak{g}_{0}$ is distinguished by the type of $\mathfrak{g}$ (or its compact real form $\mathfrak{u}_{0}$ ) and the integer $\operatorname{dim} \mathfrak{k}_{0}-\operatorname{dim} \mathfrak{p}_{0}$. For example, the notation $\mathfrak{e}_{6,-2}$ (written by Helgason as $\mathfrak{e}_{6(2)}$, as he used the integer $\operatorname{dim} \mathfrak{p}_{0}-\operatorname{dim} \mathfrak{k}_{0}$ instead) means the compact real form of the complexified Lie algebra has type $\mathfrak{e}_{6}$ and $\operatorname{dim} \mathfrak{k}_{0}-\operatorname{dim} \mathfrak{p}_{0}=-2$. 
The elements (coroots) $H_{i}^{\prime}$ are defined in (2) and (5).

i) Type A. For $\mathfrak{u}_{0}=\mathfrak{s u}(n), n \geq 3,\left\{\operatorname{Ad}\left(I_{p, n-p}\right) \mid 1 \leq p \leq n / 2\right\}$ (type AIII), $\{\tau=$ complex conjugation $\}$ (type $\left.\mathbf{A I}),\left\{\tau \circ \operatorname{Ad}\left(J_{n / 2}\right)\right\}\right\}$ (type AII) represent all conjugacy classes of involutions in $\operatorname{Aut}\left(\mathfrak{u}_{0}\right)$. The corresponding real forms are $\mathfrak{s u}(p, n-p), \mathfrak{s l}(n, \mathbb{R}), \mathfrak{s l}\left(\frac{n}{2}, \mathbb{U}\right)$.

ii) Type B. For $\mathfrak{u}_{0}=\mathfrak{s o}(2 n+1), n \geq 1,\left\{\operatorname{Ad}\left(I_{p, 2 n+1-p}\right) \mid 1 \leq p \leq n\right\}$ (type BI) represent all conjugacy classes of involutions in Aut $\left(\mathfrak{u}_{0}\right)$. The corresponding real forms are $\mathfrak{s o}(p, 2 n+1-p)$.

iii) Type C. For $\mathfrak{u}_{0}=\mathfrak{s p}(n), n \geq 3,\left\{\operatorname{Ad}\left(I_{p, n-p}\right) \mid 1 \leq p \leq n / 2\right\}$ (type CII) and $\{\operatorname{Ad}(\mathbf{i} I)\}$ (type $\mathbf{C I})$ represent all conjugacy classes of involutions in $\operatorname{Aut}\left(\mathfrak{u}_{0}\right)$. The corresponding real forms are $\mathfrak{s p}(p, n-p), \mathfrak{s p}(n, \mathbb{R})$.

iv) Type D. For $\mathfrak{u}_{0}=\mathfrak{s o}(2 n), n \geq 4,\left\{\operatorname{Ad}\left(I_{p, 2 n-p}\right) \mid 1 \leq p \leq n\right\}$ (type DI) and $\left\{\operatorname{Ad}\left(J_{n}\right)\right\}$ (type DIII) represent all conjugacy classes of involutions in $\operatorname{Aut}\left(\mathfrak{u}_{0}\right)$. The corresponding real forms are $\mathfrak{s o}(p, 2 n-p), \mathfrak{s o}^{*}(2 n, \mathbb{R}) .^{2}$

v) Type $\mathbf{E}_{\mathbf{6}}$. For $\mathfrak{u}_{0}=\mathfrak{e}_{6}$, let $\tau$ be a specific diagram involution defined by

$$
\begin{array}{cccc}
\tau\left(H_{\alpha_{1}}\right)=H_{\alpha_{6}}, & \tau\left(H_{\alpha_{6}}\right)=H_{\alpha_{1}}, & \tau\left(H_{\alpha_{3}}\right)=H_{\alpha_{5}}, \\
\tau\left(H_{\alpha_{5}}\right)=H_{\alpha_{3}}, & \tau\left(H_{\alpha_{2}}\right)=H_{\alpha_{2}}, & \tau\left(H_{\alpha_{4}}\right)=H_{\alpha_{4}}, \\
\tau\left(X_{ \pm \alpha_{1}}\right)=X_{ \pm \alpha_{6}}, & \tau\left(X_{ \pm \alpha_{6}}\right)=X_{ \pm \alpha_{1}}, & \tau\left(X_{ \pm \alpha_{3}}\right)=X_{ \pm \alpha_{5}}, \\
\tau\left(X_{ \pm \alpha_{5}}\right)=X_{ \pm \alpha_{3}}, & \tau\left(X_{ \pm \alpha_{2}}\right)=X_{ \pm \alpha_{2}}, & \tau\left(X_{ \pm \alpha_{4}}\right)=X_{ \pm \alpha_{4}} .
\end{array}
$$

Let $\sigma_{1}=\exp \left(\pi i H_{2}^{\prime}\right), \sigma_{2}=\exp \left(\pi i\left(H_{1}^{\prime}+H_{6}^{\prime}\right)\right), \sigma_{3}=\tau, \sigma_{4}=\tau \exp \left(\pi i H_{2}^{\prime}\right)$. Then $\sigma_{1}, \sigma_{2}, \sigma_{3}, \sigma_{4}$ represent all conjugacy classes of involutions in $\operatorname{Aut}\left(\mathfrak{u}_{0}\right)$, which correspond to Riemannian symmetric pairs of type EII, EIII, EIV, EI and the corresponding real forms are $\mathfrak{e}_{6,-2}, \mathfrak{e}_{6,14}, \mathfrak{e}_{6,26}, \mathfrak{e}_{6,-6}$. Also, $\sigma_{1}, \sigma_{2}$ are inner automorphisms and $\sigma_{3}, \sigma_{4}$ are outer automorphisms.

vi) Type $\mathbf{E}_{7}$. For $\mathfrak{u}_{0}=\mathfrak{e}_{7}$, let

$$
\begin{aligned}
& \sigma_{1}=\exp \left(\pi i H_{2}^{\prime}\right), \\
& \sigma_{2}=\exp \left(\pi i \frac{H_{2}^{\prime}+H_{5}^{\prime}+H_{7}^{\prime}}{2}\right), \\
& \sigma_{3}=\exp \left(\pi i \frac{H_{2}^{\prime}+H_{5}^{\prime}+H_{7}^{\prime}+2 H_{1}^{\prime}}{2}\right) .
\end{aligned}
$$

Then $\sigma_{1}, \sigma_{2}, \sigma_{3}$ represent all conjugacy classes of involutions in $\operatorname{Aut}\left(\mathfrak{u}_{0}\right)$, which correspond to Riemannian symmetric pairs of type EVI, EVII, EV and the corresponding real forms are $\mathfrak{e}_{7,5}, \mathfrak{e}_{7,25}, \mathfrak{e}_{7,-7}$

vii) Type $\mathbf{E}_{\mathbf{8}}$. For $\mathfrak{u}_{0}=\mathfrak{e}_{8}$, let

$$
\sigma_{1}=\exp \left(\pi i H_{2}^{\prime}\right), \quad \sigma_{2}=\exp \left(\pi i\left(H_{2}^{\prime}+H_{1}^{\prime}\right)\right) .
$$

${ }^{2}$ When $n=4$, we have $\operatorname{Ad}\left(I_{2,6}\right) \sim \operatorname{Ad}\left(J_{4}\right)$, and $\mathfrak{s o}(2,6) \cong \mathfrak{s o *}(8)$. 
Then $\sigma_{1}, \sigma_{2}$ represent all conjugacy classes of involutions in $\operatorname{Aut}\left(\mathfrak{u}_{0}\right)$, which correspond to Riemannian symmetric pairs of type EIX, EVIII and the corresponding real forms are $\mathfrak{e}_{8,24}, \mathfrak{e}_{8,-8}$.

viii) Type $\mathbf{F}_{\mathbf{4}}$. For $\mathfrak{u}_{0}=\mathfrak{f}_{4}$, let

$$
\sigma_{1}=\exp \left(\pi i H_{1}^{\prime}\right), \quad \sigma_{2}=\exp \left(\pi i H_{4}^{\prime}\right) .
$$

Then $\sigma_{1}, \sigma_{2}$ represent all conjugacy classes of involutions in $\operatorname{Aut}\left(\mathfrak{u}_{0}\right)$, which correspond to Riemannian symmetric pairs of type FI, FII and the corresponding real forms are $\mathfrak{f}_{4,-4}, \mathfrak{f}_{4,20}$.

ix) Type $\mathbf{G}_{2}$. For $\mathfrak{u}_{0}=\mathfrak{g}_{2}$, let $\sigma=\exp \left(\pi H_{1}^{\prime}\right)$, which represents the unique conjugacy class of involutions in $\operatorname{Aut}\left(\mathfrak{u}_{0}\right)$ and corresponds to a Riemannian symmetric pair of type $\mathbf{G}$ and the corresponding real form is $\mathfrak{g}_{2,-2}$.

\section{Centralizer of an automorphism}

In this section we prove a property of the centralizer $G^{x}$ of an element $x$ in a complex or compact Lie group $G$. First, we recall a theorem of Steinberg [Carter 1993, pp. 93-95].

Proposition 4.1 (Steinberg). Let $G$ be a connected and simply connected semisimple complex (or compact) Lie group. Then the centralizer $G^{x}$ for any $x \in G$ is connected.

For an element $x$ in a group, we write $o(x)$ for the order of $x$. The notation

$$
\operatorname{Int}(\mathfrak{g})_{0}^{\theta}
$$

in this paper always means $\left(\operatorname{Int}(\mathfrak{g})^{\theta}\right)_{0}$, not $\left(\operatorname{Int}(\mathfrak{g})_{0}\right)^{\theta}$. Similarly for

$$
\operatorname{Int}\left(\mathfrak{u}_{0}\right)_{0}^{\theta}, \operatorname{Aut}\left(\mathfrak{u}_{0}\right)_{0}^{\theta}, \operatorname{Aut}(\mathfrak{g})_{0}^{\theta} .
$$

Proposition 4.2. Let $\mathfrak{g}$ be a complex simple Lie algebra. Suppose that the order of an element $\theta \in \operatorname{Aut}(\mathfrak{g})$ is equal to the order of the coset element $\theta \operatorname{Int}(\mathfrak{g})$ in $\operatorname{Out}(\mathfrak{g})=\operatorname{Aut}(\mathfrak{g}) / \operatorname{Int}(\mathfrak{g})$, that is, $o(\theta)=o(\theta \operatorname{Int}(\mathfrak{g}))$. Then $Z_{\operatorname{Int}(\mathfrak{g})}\left(\operatorname{Int}(\mathfrak{g})_{0}^{\theta}\right)=1$.

Proof. By the assumption, $\theta$ is a diagram automorphism; this means there exists a Cartan subalgebra $\mathfrak{t}$ which is stable under $\theta$ and $\theta$ maps $\Delta^{+}$to itself, where $\Delta=\Delta(\mathfrak{g}, \mathfrak{t})$ and $\Delta^{+}$is a positive system. For any $\alpha \in \Delta$, let $\theta\left(X_{\alpha}\right)=a_{\alpha} X_{\theta \alpha}$ with $a_{\alpha} \neq 0$.

Let $k=o(\theta)=o(\theta \operatorname{Int}(\mathfrak{g}))$. Then, for any $\alpha \in \Delta$,

$$
X_{\alpha}=\theta^{k}\left(X_{\alpha}\right)=\left(\prod_{0 \leq j \leq k-1} a_{\theta^{j} \alpha}\right) X_{\theta^{k} \alpha} .
$$


It follows that

$$
\prod_{0 \leq j \leq k-1} a_{\theta^{j} \alpha}=1
$$

Let $L=\operatorname{Int}(\mathfrak{g})_{0}^{\theta}, \mathfrak{s}=\mathfrak{t}^{\theta}, T=\exp (\operatorname{ad} \mathfrak{t})$ and $S=\exp (\operatorname{ad} \mathfrak{s})$. It is clear that $S \subset L$. We first show that $Z_{\operatorname{Int}(\mathfrak{g})}(S)=T$. It is clear that $\mathfrak{t} \subset Z_{\mathfrak{g}}(\mathfrak{s})$. Suppose that $X_{\alpha} \in Z_{\mathfrak{g}}(\mathfrak{s})$ for some $\alpha \in \Delta^{+}$. Since $\theta^{k}=1$, we have $\sum_{0 \leq j \leq k-1} \theta^{j}(H) \in \mathfrak{t}^{\theta}=\mathfrak{s}$ for any $H \in \mathfrak{t}$. Then $\left[\sum_{0 \leq j \leq k-1} \theta^{j}(H), X_{\alpha}\right]=0$.

For any $j$, we have

$$
\begin{aligned}
{\left[\theta^{j} H, X_{\alpha}\right] } & =\theta^{j}\left(\left[H, \theta^{k-j} X_{\alpha}\right]\right)=\theta^{j}\left(\left(\prod_{0 \leq i \leq k-j-1} a_{\theta^{i} \alpha}\right) \cdot\left(\left(\theta^{k-j} \alpha\right) H\right) \cdot X_{\theta^{k-j} \alpha}\right) \\
& =\left(\prod_{0 \leq i \leq k-j-1} a_{\theta^{i} \alpha}\right) \cdot\left(\left(\theta^{k-j} \alpha\right) H\right) \cdot\left(\prod_{0 \leq i \leq j-1} a_{\theta^{k-j+i} \alpha}\right) X_{\alpha} \\
& =\left(\prod_{0 \leq i \leq k-1} a_{\theta^{i} \alpha}\right) \cdot\left(\left(\theta^{k-j} \alpha\right) H\right) \cdot X_{\alpha}=\left(\left(\theta^{k-j} \alpha\right) H\right) \cdot X_{\alpha} .
\end{aligned}
$$

Hence $0=\left[\sum_{0 \leq j \leq k-1} \theta^{j}(H), X_{\alpha}\right]=\left(\left(\sum_{0 \leq j \leq k-1} \theta^{k-j} \alpha\right) H\right) \cdot X_{\alpha}$. This implies

$$
\sum_{0 \leq j \leq k-1} \theta^{j} \alpha=0
$$

which contradicts that all $\theta^{j} \alpha$ are positive roots. So $Z_{\mathfrak{g}}(\mathfrak{s})=\mathfrak{t}$. Since $Z_{\operatorname{Int}(\mathfrak{g})}(S)$ is connected (by Corollary 4.51 of [Knapp 2002, p. 260], which also applies to complex semisimple groups), $Z_{\operatorname{Int}(\mathfrak{g})}(S)=T$.

Now we show that $Z_{\text {Int }(\mathfrak{g})}(L)=1$. Suppose that $1 \neq \tau \in Z_{\text {Int }(\mathfrak{g})}(L)$. By the above, we have $Z_{\text {Int }(\mathfrak{g})}(L) \subset Z_{\operatorname{Int}(\mathfrak{g})}(S)=T$, then $\tau=\exp (\operatorname{ad} H)$ for some $H \in \mathfrak{t}$. For any $\alpha \in \Delta, \sum_{0 \leq j \leq k-1} \theta^{j}\left(X_{\alpha}\right) \in \mathfrak{g}^{\theta}$ (since $\theta^{k}=1$ ), so

$\sum_{0 \leq j \leq k-1} \theta^{j}\left(X_{\alpha}\right)=\tau\left(\sum_{0 \leq j \leq k-1} \theta^{j}\left(X_{\alpha}\right)\right)=\sum_{0 \leq j \leq k-1} \tau\left(\theta^{j}\left(X_{\alpha}\right)\right)=\sum_{0 \leq j \leq k-1} e^{\left(\theta^{j} \alpha\right) H} \theta^{j}\left(X_{\alpha}\right)$.

Since each $\theta^{j}\left(X_{\alpha}\right)$ is of the form $\theta^{j}\left(X_{\alpha}\right)=b_{j} X_{\theta^{j} \alpha}$ for some $b_{j} \neq 1$, the last equality implies $\tau\left(X_{\alpha}\right)=X_{\alpha}$ if $\left\{\theta^{j} \alpha, 0 \leq j \leq k-1\right\}$ are distinct.

Claim 4.3. Those $\alpha \in \Delta$ with roots in $\left\{\theta^{j} \alpha, 0 \leq j \leq k-1\right\}$ pairwise different generate $\Delta$ (as a root system).

Since $\tau\left(X_{\alpha}\right)=X_{\alpha}$ when the elements $\theta^{j} \alpha$ are distinct for $0 \leq j \leq k-1$, by Claim 4.3, we have $\tau\left(X_{\alpha}\right)=X_{\alpha}$ for any $\alpha \in \Delta$. Hence $\tau=1$, which is to say, $Z_{\operatorname{Int}(\mathfrak{g})}\left(\operatorname{Int}(\mathfrak{g})_{0}^{\theta}\right)=1$. 
Proof of Claim 4.3. Note that $\theta$ maps $\Delta^{+}$to itself, so it maps the simple system $\Pi=\left\{\alpha_{1}, \ldots, \alpha_{r}\right\}$ to itself. We have four cases to consider, that is, $\Delta=\mathrm{A}_{n}(n \geq 2)$, $\mathrm{D}_{n}(n \geq 4), \mathrm{E}_{6}$ and $\theta$ is an automorphism of order 2 , or $\Delta=\mathrm{D}_{4}$ and $\theta$ is an automorphism of order 3 . We give the proof when $\Delta=\mathrm{A}_{2 n}(n \geq 1)$ and $o(\theta)=2$. The proof for other cases is similar.

When $\Delta=\mathrm{A}_{2 n}(n \geq 1)$ and $o(\theta)=2$, we have $\theta\left(\alpha_{i}\right)=\alpha_{2 n+1-i}$ and $\theta\left(\alpha_{2 n+1-i}\right)=$ $\alpha_{i}$ for any $i, 1 \leq i \leq n$. For $1 \leq i \leq n$, let

$$
\beta_{i}=\sum_{1 \leq j \leq i} \alpha_{j} \quad \text { and } \quad \beta_{i}^{\prime}=\sum_{1 \leq j \leq i} \alpha_{2 n+1-j} .
$$

Then $\theta\left( \pm \beta_{i}\right) \neq \pm \beta_{i}, \theta\left( \pm \beta_{i}^{\prime}\right) \neq \pm \beta_{i}^{\prime}$ and $\left\{ \pm \beta_{i}, \pm \beta_{i}^{\prime}: 1 \leq i \leq n\right\}$ generate $\Delta$.

Corollary 4.4. Let $\mathfrak{u}_{0}$ be a compact simple Lie algebra. If $\theta \in \operatorname{Aut}\left(\mathfrak{u}_{0}\right)$ satisfies the condition $o(\theta)=o\left(\theta \operatorname{Int}\left(\mathfrak{u}_{0}\right)\right)$, then $Z_{\operatorname{Int}\left(\mathfrak{u}_{0}\right)}\left(\operatorname{Int}\left(\mathfrak{u}_{0}\right)_{0}^{\theta}\right)=1$.

Corollary 4.4 indicates that if $G$ is a compact (simple) Lie group of adjoint type and $x$ is of minimal possible order among all elements in the connected component containing it, then $\left(G^{x}\right)_{0}$ is also of adjoint type and the conjugation action of any element $y \in G^{x}-\left(G^{x}\right)_{0}$ on $\left(G^{x}\right)_{0}$ is an outer automorphism.

\section{Symmetric subgroups of $\operatorname{Aut}\left(\mathfrak{u}_{0}\right)$}

Let $\mathfrak{u}_{0}$ be a compact simple Lie algebra. For each conjugacy class of involutions in $\operatorname{Aut}\left(\mathfrak{u}_{0}\right)$, we choose a representative $\theta$ as in Section 3 and determine the symmetric subgroup $\operatorname{Aut}\left(\mathfrak{u}_{0}\right)^{\theta}$.

When $\mathfrak{u}_{0}$ is a classical simple Lie algebra nonisomorphic to $\mathfrak{s o}(8)$ or $\mathfrak{u}_{0}=\mathfrak{s o}(8)$ but $\theta \nsim \operatorname{Ad}\left(I_{4,4}\right)$, we can use matrices to represent involutions $\theta$ and calculate the corresponding $\operatorname{Aut}\left(\mathfrak{u}_{0}\right)^{\theta}$. In the case of $\theta=\operatorname{Ad}\left(I_{4,4}\right) \in \operatorname{Aut}(\mathfrak{s o}(8))$, we have $\theta \sim \exp \left(\pi i H_{2}^{\prime}\right)$. Then

$$
\operatorname{Int}(\mathfrak{s o}(8))^{\theta}=\left(\operatorname{Sp}(1)^{4} / Z^{\prime}\right) \rtimes D,
$$

where $Z^{\prime}=\left\{\left(\epsilon_{1}, \epsilon_{2}, \epsilon_{3}, \epsilon_{4}\right) \mid \epsilon_{i}= \pm 1, \epsilon_{1} \epsilon_{2} \epsilon_{3} \epsilon_{4}=1\right\}$, and $D \subset S_{4}$ is the (unique) normal order four subgroup of $S_{4}$ with conjugation action on $\left(\operatorname{Sp}(1)^{4}\right) / Z^{\prime}$ by permutations. Then we observe that there exists a subgroup of Aut $(\mathfrak{s o}(8))$ that projects isomorphically to $\operatorname{Aut}(\mathfrak{s o}(8)) / \operatorname{Int}(\mathfrak{s o}(8)) \cong S_{3}$ and is contained in $\operatorname{Aut}(\mathfrak{s o}(8))^{\theta}$. A little more argument shows

$$
\operatorname{Aut}(\mathfrak{s o}(8))^{\theta}=\left(\operatorname{Sp}(1)^{4} / Z^{\prime}\right) \rtimes S_{4} .
$$

When $\mathfrak{u}_{0}$ is an exceptional simple Lie algebra, we first determine the symmetric subalgebra $\mathfrak{k}_{0}=\mathfrak{u}_{0}^{\theta}$ and the highest weights of the isotropic space $\mathfrak{p}_{0}=\mathfrak{u}_{0}^{-\theta}$ as a $\mathfrak{k}_{0}$-module. The results are summarized in Table 1 . The coroots $H_{i}^{\prime}$ are defined in (2) and (5) and the involutions are defined in Section 3. 


\begin{tabular}{|cccc|}
\hline & $\theta$ & $\mathfrak{k}_{0}$ & $\mathfrak{p}$ \\
\hline EI & $\sigma_{4}=\tau \exp \left(\pi i H_{2}^{\prime}\right)$ & $\mathfrak{s p}(4)$ & $V_{\omega_{4}}$ \\
EII & $\sigma_{1}=\exp \left(\pi i H_{2}^{\prime}\right)$ & $\mathfrak{s u}(6) \oplus \mathfrak{s p}(1)$ & $\bigwedge^{3} \mathbb{C}^{6} \otimes \mathbb{C}^{2}$ \\
$\mathbf{E I I I}$ & $\sigma_{2}=\exp \left(\pi i\left(H_{1}^{\prime}+H_{6}^{\prime}\right)\right)$ & $\mathfrak{s o}(10) \oplus i \mathbb{R}$ & $\left(M_{+} \otimes 1\right) \oplus\left(M_{-} \otimes \overline{1}\right)$ \\
$\mathbf{E I V}$ & $\sigma_{3}=\tau$ & $\mathfrak{f}_{4}$ & $V_{\omega_{4}}$ \\
$\mathbf{E V}$ & $\sigma_{3}=\exp \left(\pi i\left(H_{1}^{\prime}+H_{0}^{\prime}\right)\right)$ & $\mathfrak{s u}(8)$ & $\wedge^{4} \mathbb{C}^{8}$ \\
EVI & $\sigma_{1}=\exp \left(\pi i H_{2}^{\prime}\right)$ & $\mathfrak{s o}(12) \oplus \mathfrak{s p}(1)$ & $M_{+} \otimes \mathbb{C}^{2}$ \\
EVII & $\sigma_{2}=\exp \left(\pi i H_{0}^{\prime}\right)$ & $\mathfrak{e}_{6} \oplus i \mathbb{R}$ & $\left(V_{\omega_{1}} \otimes 1\right) \oplus\left(V_{\omega_{6}} \otimes \overline{1}\right)$ \\
$\mathbf{E V I I I}$ & $\sigma_{2}=\exp \left(\pi i\left(H_{1}^{\prime}+H_{2}^{\prime}\right)\right)$ & $\mathfrak{s o}(16)$ & $M_{+}$ \\
$\mathbf{E I X}$ & $\sigma_{1}=\exp \left(\pi i H_{1}^{\prime}\right)$ & $\mathfrak{e}_{7} \oplus \mathfrak{s p}(1)$ & $V_{\omega_{7}} \otimes \mathbb{C}^{2}$ \\
FI & $\sigma_{1}=\exp \left(\pi i H_{1}^{\prime}\right)$ & $\mathfrak{s p}(3) \oplus \mathfrak{s p}(1)$ & $V_{\omega_{3}} \otimes \mathbb{C}^{2}$ \\
FII & $\sigma_{2}=\exp \left(\pi i H_{4}^{\prime}\right)$ & $\mathfrak{s o}(9)$ & $M$ \\
G & $\sigma=\exp \left(\pi i H_{1}^{\prime}\right)$ & $\mathfrak{s p}(1) \oplus \mathfrak{s p}(1)$ & $\operatorname{Sym}^{3} \mathbb{C}^{2} \otimes \mathbb{C}^{2}$ \\
\hline
\end{tabular}

Table 1. Symmetric pairs and isotropic modules (exceptional Lie algebras case).

Since any element of $\operatorname{Aut}\left(\mathfrak{u}_{0}\right)^{\theta}$ which acts trivially on both $\mathfrak{k}_{0}$ and $\mathfrak{p}_{0}$ must be trivial, the isomorphism type of $\mathfrak{k}_{0}$ and its isotropic module $\mathfrak{p}$ determine $\operatorname{Aut}\left(\mathfrak{u}_{0}\right)_{0}^{\theta}$ completely. We may get $\operatorname{Aut}\left(\mathfrak{u}_{0}\right)_{0}^{\theta}$ in the following way. Start with a compact connected Lie group $H$ of the form $H=A \times H_{s}$ with $A=Z\left(\operatorname{Aut}\left(\mathfrak{u}_{0}\right)_{0}^{\theta}\right)_{0}$ a connected torus $\left(A \cong \mathrm{U}(1)^{s}\right.$ with $\left.s=\operatorname{dim} \mathfrak{z}\left(\mathfrak{k}_{0}\right)\right)$ and $H_{s}$ a connected and simply connected compact Lie group with Lie $H_{s}=\left[\mathfrak{k}_{0}, \mathfrak{k}_{0}\right]$ (then Lie $H=\mathfrak{k}_{0}=\mathfrak{u}_{0}^{\theta}$ ). Then we have a surjective homomorphism

$$
\pi: H \rightarrow \operatorname{Aut}\left(\mathfrak{u}_{0}\right)
$$

determined by $\mathfrak{g}$ as a $\mathfrak{k}_{0}$-module. With this construction, it is clear that $\operatorname{Im}(\pi)=$ $\operatorname{Aut}\left(\mathfrak{u}_{0}\right)_{0}^{\theta}$ and ker $\pi$ is determined by $\mathfrak{k}_{0}$ and its module $\mathfrak{p}$ (as described in Table 1 ). By Proposition 4.1 and Corollary 4.4, we can also determine the number of connected components of $\operatorname{Aut}\left(\mathfrak{u}_{0}\right)^{\theta}$. Then we could find elements outside $\operatorname{Aut}\left(\mathfrak{u}_{0}\right)_{0}^{\theta}$ to generate $\operatorname{Aut}\left(\mathfrak{u}_{0}\right)^{\theta}$ together with $\operatorname{Aut}\left(\mathfrak{u}_{0}\right)_{0}^{\theta}$. We show the detailed argument in most cases below. The results about the symmetric subgroups $\operatorname{Aut}\left(\mathfrak{u}_{0}\right)^{\theta}$ are given in the last column of Table 2. The information about the first three columns of Table 2 is contained in [Knapp 2002, pp. 408-426]. The fourth column is from Section 3.

5A. Type $\mathbf{E}_{6}$. Now $\mathfrak{u}_{0}=\mathfrak{e}_{6}$. Consider an outer automorphism $\theta=\sigma_{3}$ or $\sigma_{4}$. By Corollary 4.4, any element in $\operatorname{Int}\left(\mathfrak{u}_{0}\right)^{\theta}-\operatorname{Aut}\left(\mathfrak{u}_{0}\right)_{0}^{\theta}$ acts on $\mathfrak{u}_{0}^{\theta}$ as an outer automorphism. Note that $\mathfrak{u}_{0}^{\theta} \cong \mathfrak{s p}(4)$ or $\mathfrak{f}_{4}$, so it has no outer automorphisms. By Corollary 4.4, it follows that $\operatorname{Int}\left(\mathfrak{u}_{0}\right)^{\theta}=\operatorname{Aut}\left(\mathfrak{u}_{0}\right)_{0}^{\theta}$ and $\operatorname{Aut}\left(\mathfrak{u}_{0}\right)^{\theta}=\operatorname{Aut}\left(\mathfrak{u}_{0}\right)_{0}^{\theta} \times\langle\theta\rangle$. Moreover, $\operatorname{Aut}\left(\mathfrak{u}_{0}\right)_{0}^{\theta}$ is of adjoint type by Corollary 4.4. 


\begin{tabular}{|c|c|c|c|c|}
\hline Type & $\left(\mathfrak{u}_{0}, \mathfrak{k}_{0}\right)$ & rank & $\theta$ & symmetric subgroup Aut $\left(\mathfrak{u}_{0}\right)^{\theta}$ \\
\hline AI & $(\mathfrak{s u}(n), \mathfrak{s o}(n))$ & $n-1$ & $\bar{X}$ & $(O(n) /\langle-I\rangle) \times\langle\theta\rangle$ \\
\hline AII & $(\mathfrak{s u}(2 n), \mathfrak{s p}(n))$ & $n-1$ & $J_{n} \bar{X} J_{n}^{-1}$ & $(\operatorname{Sp}(n) /\langle-I\rangle) \times\langle\theta\rangle$ \\
\hline $\begin{array}{l}\text { AIII } \\
p<q\end{array}$ & $(\mathfrak{s u}(p+q), \mathfrak{s}(\mathfrak{u}(p)+\mathfrak{u}(q)))$ & & $I_{p, q} X I_{p, q}$ & $\begin{array}{c}\left(S(U(p) \times U(q)) / Z_{p+q}\right) \rtimes\langle\tau\rangle \\
\operatorname{Ad}(\tau)=\text { complex conjugation }\end{array}$ \\
\hline $\begin{array}{l}\text { AIII } \\
p=q\end{array}$ & $(\mathfrak{s u}(2 p), \mathfrak{s}(\mathfrak{u}(p)+\mathfrak{u}(p)))$ & $p$ & ${ }_{p, p} X I_{p, p}$ & $\begin{array}{c}\left(S(U(p) \times U(p)) / Z_{2 p}\right) \rtimes\left\langle\tau, J_{p}\right\rangle \\
\operatorname{Ad}\left(J_{p}\right)(X, Y)=(Y, X)\end{array}$ \\
\hline $\begin{array}{l}\text { BDI } \\
p<q\end{array}$ & $(\mathfrak{s o}(p+q), \mathfrak{s o}(p)+\mathfrak{s o}(q))$ & $p$ & $I_{p, q} X I_{p, q}$ & $(O(p) \times O(q)) /\left\langle\left(-I_{p},-I_{q}\right)\right\rangle$ \\
\hline $\begin{array}{c}\text { DI } \\
p>4\end{array}$ & $(\mathfrak{s o}(2 p), \mathfrak{s o}(p)+\mathfrak{s o}(p))$ & $p$ & $I_{p, p} X I_{p, p}$ & $\begin{array}{c}\left((O(p) \times O(p)) /\left\langle\left(-I_{p},-I_{p}\right)\right\rangle\right) \rtimes\left\langle J_{p}\right\rangle \\
\operatorname{Ad}\left(J_{p}\right)(X, Y)=(Y, X)\end{array}$ \\
\hline $\begin{array}{c}\text { DI } \\
p=4\end{array}$ & $(\mathfrak{s o}(8), \mathfrak{s o}(4)+\mathfrak{s o}(4))$ & 4 & $I_{4,4} X I_{4,4}$ & $\begin{array}{c}\quad\left(\left(\operatorname{Sp}(1)^{4}\right) / Z^{\prime}\right) \rtimes S_{4} \\
S_{4} \text { acts by permutations }\end{array}$ \\
\hline DIII & $(\mathfrak{s o}(2 n), \mathfrak{u}(n))$ & $n$ & $J_{n} X J_{n}^{-1}$ & $\begin{array}{c}(U(n) /\{ \pm I\}) \rtimes\left\langle I_{n, n}\right\rangle \\
\operatorname{Ad}\left(I_{n, n}\right)=\text { complex conjugation }\end{array}$ \\
\hline CI & $(\mathfrak{s p}(n), \mathfrak{u}(n))$ & $n(\mathbf{i}$ & $I) X(\mathbf{i} I)^{-1}$ & $\begin{array}{c}(U(n) /\{ \pm I\}) \rtimes\langle\mathbf{j} I\rangle \\
\operatorname{Ad}(\mathbf{j} I)=\text { complex conjugation }\end{array}$ \\
\hline $\begin{array}{c}\text { CII } \\
p<q\end{array}$ & $(\mathfrak{s p}(p+q), \mathfrak{s p}(p)+\mathfrak{s p}(q))$ & $p$ & $I_{p, q} X I_{p, q}$ & $(\operatorname{Sp}(p) \times \operatorname{Sp}(q)) /\left\langle\left(-I_{p},-I_{q}\right)\right\rangle$ \\
\hline $\begin{array}{c}\text { CII } \\
p=q\end{array}$ & $(\mathfrak{s p}(2 p), \mathfrak{s p}(p)+\mathfrak{s p}(p))$ & $p$ & $I_{p, p} X I_{p, p}$ & $\begin{array}{c}\left(\left(\operatorname{Sp}(p) \times \operatorname{Sp}(p) /\left\langle\left(-I_{p},-I_{p}\right)\right\rangle\right) \rtimes\left\langle J_{p}\right\rangle\right. \\
\operatorname{Ad}\left(J_{p}\right)(X, Y)=(Y, X)\end{array}$ \\
\hline EI & $\left(\mathfrak{e}_{6}, \mathfrak{s p}(4)\right)$ & 6 & $\sigma_{4}$ & $(\operatorname{Sp}(4) /\langle-1\rangle) \times\langle\theta\rangle$ \\
\hline EII & $\left(\mathfrak{e}_{6}, \mathfrak{s u}(6)+\mathfrak{s p}(1)\right)$ & 4 & $\sigma_{1} \quad(S$ & $\begin{array}{c}\left.S U(6) \times \operatorname{Sp}(1) /\left\langle\left(e^{\frac{2 \pi i}{3}} I, 1\right),(-I,-1)\right\rangle\right) \rtimes\langle\tau\rangle \\
\mathfrak{k}_{0}^{\tau}=\mathfrak{s p}(3) \oplus \mathfrak{s p}(1)\end{array}$ \\
\hline EIII & $\left(\mathfrak{e}_{6}, \mathfrak{s o}(10)+i \mathbb{R}\right)$ & 2 & $\sigma_{2}$ & $\begin{array}{c}(\operatorname{Spin}(10) \times U(1) /\langle(c, i)\rangle) \rtimes\langle\tau\rangle \\
\mathfrak{k}_{0}^{\tau}=\mathfrak{s o}(9)\end{array}$ \\
\hline EIV & $\left(\mathfrak{e}_{6}, \mathfrak{f}_{4}\right)$ & 2 & $\sigma_{3}$ & $F_{4} \times\langle\theta\rangle$ \\
\hline $\mathbf{E V}$ & $\left(\mathfrak{e}_{7}, \mathfrak{s u}(8)\right)$ & 7 & $\sigma_{3}$ & $\begin{array}{c}(S U(8) /\langle i I\rangle) \rtimes\langle\omega\rangle \\
\mathfrak{k}_{0}^{\omega}=\mathfrak{s p}(4)\end{array}$ \\
\hline EVI & $\left(\mathfrak{e}_{7}, \mathfrak{s o}(12)+\mathfrak{s p}(1)\right)$ & 4 & $\sigma_{1}$ & $(\operatorname{Spin}(12) \times \operatorname{Sp}(1)) /\langle(c, 1),(-1,-1)\rangle$ \\
\hline EVII & $\left(\mathfrak{e}_{7}, \mathfrak{e}_{6}+i \mathbb{R}\right)$ & 3 & $\sigma_{2}$ & $\begin{array}{c}\left(\left(E_{6} \times U(1)\right) /\left\langle\left(c, e^{\frac{2 \pi i}{3}}\right)\right\rangle\right) \rtimes\langle\omega\rangle \\
\mathfrak{k}_{0}^{\omega}=\mathfrak{f}_{4}\end{array}$ \\
\hline EVIII & $\left(\mathfrak{e}_{8}, \mathfrak{s o}(16)\right)$ & 8 & $\sigma_{2}$ & $\operatorname{Spin}(16) /\langle c\rangle$ \\
\hline EIX & $\left(\mathfrak{e}_{8}, \mathfrak{e}_{7}+\mathfrak{s p}(1)\right)$ & 4 & $\sigma_{1}$ & $E_{7} \times \operatorname{Sp}(1) /\langle(c,-1)\rangle$ \\
\hline FI & $\left(\mathfrak{f}_{4}, \mathfrak{s p}(3)+\mathfrak{s p}(1)\right)$ & 4 & $\sigma_{1}$ & $(\mathrm{Sp}(3) \times \mathrm{Sp}(1)) /\langle(-I,-1)\rangle$ \\
\hline FII & $\left(\mathfrak{f}_{4}, \mathfrak{s o}(9)\right)$ & 1 & $\sigma_{2}$ & $\operatorname{Spin}(9)$ \\
\hline $\mathbf{G}$ & $\left(\mathfrak{g}_{2}, \mathfrak{s p}(1)+\mathfrak{s p}(1)\right)$ & 2 & $\sigma$ & $(\operatorname{Sp}(1) \times \operatorname{Sp}(1)) /\langle(-1,-1)\rangle$ \\
\hline
\end{tabular}

Table 2. Symmetric pairs and symmetric subgroups. (When $n=4$, DIII is identical to BDI when $p=2$ and $q=6$.) 
Consider an inner automorphism $\theta=\sigma_{1}$ or $\sigma_{2}$. Let $\theta^{\prime} \in \mathrm{E}_{6}$ be an involution which maps to $\theta$ under the covering $\pi: \mathrm{E}_{6} \rightarrow \operatorname{Int}\left(\mathfrak{e}_{6}\right)$. We have

$$
\begin{aligned}
& \operatorname{Int}\left(\mathfrak{e}_{6}\right)^{\theta}=\left\{g \in \mathrm{E}_{6} \mid \theta^{\prime} g \theta^{\prime-1} g^{-1} \in Z\left(\mathrm{E}_{6}\right)\right\} / Z\left(\mathrm{E}_{6}\right), \\
& \operatorname{Int}\left(\mathfrak{e}_{6}\right)_{0}^{\theta}=\left\{g \in \mathrm{E}_{6} \mid \theta^{\prime} g \theta^{\prime-1} g^{-1}=1\right\} / Z\left(\mathrm{E}_{6}\right),
\end{aligned}
$$

(use Proposition 4.1 here). If $\left\{g \in \mathrm{E}_{6} \mid \theta^{\prime} g \theta^{-1} g^{-1} \in Z\left(\mathrm{E}_{6}\right)\right\} \neq \mathrm{E}_{6}^{\theta}$, then there exists $g \in \mathrm{E}_{6}$ such that $\theta^{\prime} g \theta^{\prime-1} g^{-1}=c \in Z\left(E_{6}\right)$. Then $g \theta^{\prime} g^{-1}=\theta^{\prime} c^{-1}$. But $o\left(\theta^{\prime}\right)=2 \neq 6=o\left(\theta^{\prime} c^{-1}\right)$. So $g \theta^{\prime} g^{-1} \neq \theta^{\prime} c^{-1}$. Then $\left\{g \in \mathrm{E}_{6} \mid \theta(g) g^{-1} \in Z\left(\mathrm{E}_{6}\right)\right\}=\mathrm{E}_{6}^{\theta}$ and so $\operatorname{Int}\left(\mathfrak{e}_{6}\right)^{\theta}=\operatorname{Int}\left(\mathfrak{e}_{6}\right)_{0}^{\theta}$. Since $\sigma_{1}, \sigma_{2}$ commutes with $\tau$,

$$
\operatorname{Aut}\left(\mathfrak{e}_{6}\right)^{\theta}=\operatorname{Int}\left(\mathfrak{e}_{6}\right)_{0}^{\theta} \rtimes\langle\tau\rangle .
$$

The conjugation action of $\tau$ on $\operatorname{Int}\left(\mathfrak{e}_{6}\right)_{0}^{\theta}$ is determined by its action on $\mathfrak{k}_{0}=\mathfrak{u}_{0}^{\theta}$, and

$$
\left(\mathfrak{e}_{6}^{\sigma_{1}}\right)^{\tau}=\mathfrak{s p}(3) \oplus \mathfrak{s p}(1), \quad\left(\mathfrak{e}_{6}^{\sigma_{2}}\right)^{\tau}=\mathfrak{s o}(9) .
$$

5B. Type $\mathbf{E}_{7}$. Now $\mathfrak{u}_{0}=\mathfrak{e}_{7}$ and $\operatorname{Aut}\left(\mathfrak{e}_{7}\right)=\operatorname{Int}\left(\mathfrak{e}_{7}\right)$ is connected. Let $\pi: \mathrm{E}_{7} \rightarrow \operatorname{Aut}\left(\mathfrak{e}_{7}\right)$ be the adjoint homomorphism, which is a 2 -fold covering. Let

$$
\begin{aligned}
& \sigma_{1}^{\prime}=\exp \left(\pi i H_{2}^{\prime}\right) \in \mathrm{E}_{7}, \\
& \sigma_{2}^{\prime}=\exp \left(\pi i \frac{H_{2}^{\prime}+H_{5}^{\prime}+H_{7}^{\prime}}{2}\right) \in \mathrm{E}_{7}, \\
& \sigma_{3}^{\prime}=\exp \left(\pi i \frac{2 H_{1}^{\prime}+H_{2}^{\prime}+H_{5}^{\prime}+H_{7}^{\prime}}{2}\right) \in \mathrm{E}_{7} .
\end{aligned}
$$

Then $\pi\left(\sigma_{i}^{\prime}\right)=\sigma_{i}, o\left(\sigma_{1}^{\prime}\right)=2, o\left(\sigma_{2}^{\prime}\right)=4$ and $o\left(\sigma_{3}^{\prime}\right)=4$. One has

$$
\begin{aligned}
& \operatorname{Aut}\left(\mathfrak{e}_{7}\right)^{\sigma_{i}} \cong\left\{g \in \mathrm{E}_{7} \mid g \sigma_{i}^{\prime} g^{-1} \sigma_{i}^{\prime-1} \in Z\left(\mathrm{E}_{7}\right)\right\} / Z\left(\mathrm{E}_{7}\right), \\
& \operatorname{Aut}\left(\mathfrak{e}_{7}\right)_{0}^{\sigma_{i}} \cong\left\{g \in \mathrm{E}_{7} \mid g \sigma_{i}^{\prime} g^{-1} \sigma_{i}^{\prime-1}=1\right\} / Z\left(\mathrm{E}_{7}\right)
\end{aligned}
$$

(use Proposition 4.1 here), where $Z\left(\mathrm{E}_{7}\right)=\left\langle\exp \left(\pi i\left(H_{2}^{\prime}+H_{5}^{\prime}+H_{7}^{\prime}\right)\right)\right\rangle \cong \mathbb{Z} / 2 \mathbb{Z}$ is the center of $\mathrm{E}_{7}$.

For $\theta=\sigma_{1}$, suppose that there exists $g \in \mathrm{E}_{7}$ such that

$$
g \sigma_{1}^{\prime} g^{-1}\left(\sigma_{1}^{\prime}\right)^{-1}=\exp \left(\pi i\left(H_{2}^{\prime}+H_{5}^{\prime}+H_{7}^{\prime}\right)\right) .
$$

Then $g \exp \left(\pi i H_{2}^{\prime}\right) g^{-1}=\exp \left(\pi i\left(H_{5}^{\prime}+H_{7}^{\prime}\right)\right)$. Then there exists $w \in W$ such that $w\left(\exp \left(\pi i H_{2}^{\prime}\right)\right)=\exp \left(\pi i\left(H_{5}^{\prime}+H_{7}^{\prime}\right)\right)$. Since $w\left(\exp \left(\pi i H_{\alpha_{2}}^{\prime}\right)\right)=\exp \left(\pi i H_{w\left(\alpha_{2}\right)}^{\prime}\right)$, we get $\exp \left(\pi i H_{w\left(\alpha_{2}\right)}^{\prime}\right)=\exp \left(\pi i\left(H_{5}^{\prime}+H_{7}^{\prime}\right)\right)$. Then

$$
w\left(\alpha_{2}\right) \in\left(\alpha_{5}+\alpha_{7}\right)+2 \operatorname{span}_{\mathbb{Z}}\left\{\alpha_{1}, \alpha_{2}, \alpha_{3}, \alpha_{4}, \alpha_{5}, \alpha_{6}, \alpha_{7}\right\} .
$$

There are no roots in $\left(\alpha_{5}+\alpha_{7}\right)+2 \operatorname{span}_{\mathbb{Z}}\left\{\alpha_{1}, \alpha_{2}, \alpha_{3}, \alpha_{4}, \alpha_{5}, \alpha_{6}, \alpha_{7}\right\}$, so there are no $g \in \mathrm{E}_{7}$ such that $\left(g \sigma_{1}^{\prime} g^{-1}\right) \sigma_{1}^{\prime-1}=\exp \left(\pi i\left(H_{2}^{\prime}+H_{5}^{\prime}+H_{7}^{\prime}\right)\right)$. Then

$$
\left\{g \in \mathrm{E}_{7} \mid\left(g \sigma_{1}^{\prime} g^{-1}\right) \sigma_{1}^{\prime-1} \in Z\left(\mathrm{E}_{7}\right)\right\}=\mathrm{E}_{7}^{\sigma_{1}^{\prime}} .
$$


So $\operatorname{Aut}\left(\mathfrak{e}_{7}\right)^{\sigma_{1}}=\operatorname{Aut}\left(\mathfrak{e}_{7}\right)_{0}^{\sigma_{1}}$.

For $\theta=\sigma_{2}$ or $\sigma_{3}$, let

$$
\omega=\exp \left(\frac{\pi\left(X_{\alpha_{2}}-X_{-\alpha_{2}}\right)}{2}\right) \exp \left(\frac{\pi\left(X_{\alpha_{5}}-X_{-\alpha_{5}}\right)}{2}\right) \exp \left(\frac{\pi\left(X_{\alpha_{7}}-X_{-\alpha_{7}}\right)}{2}\right) .
$$

Then

$$
\begin{aligned}
& \omega \sigma_{2}^{\prime} \omega^{-1}=\sigma_{2}^{\prime-1}=\sigma_{2}^{\prime} \exp \left(\pi i\left(H_{2}^{\prime}+H_{5}^{\prime}+H_{7}^{\prime}\right)\right), \\
& \omega \sigma_{3}^{\prime} \omega^{-1}=\sigma_{3}^{\prime-1}=\sigma_{3}^{\prime} \exp \left(\pi i\left(H_{2}^{\prime}+H_{5}^{\prime}+H_{7}^{\prime}\right)\right),
\end{aligned}
$$

and $\omega^{2}=1$. Then $\operatorname{Aut}\left(\mathfrak{e}_{7}\right)^{\theta}=\operatorname{Aut}\left(\mathfrak{e}_{7}\right)_{0}^{\theta} \rtimes\langle\omega\rangle$. The conjugation action of $\omega$ on $\operatorname{Aut}\left(\mathfrak{e}_{7}\right)_{0}^{\theta}$ is determined by its action on $\mathfrak{k}_{0}=\mathfrak{u}_{0}^{\theta}$, and we have

$$
\left(\mathfrak{e}_{7}^{\sigma_{2}}\right)^{\omega}=\mathfrak{f}_{4}, \quad\left(\mathfrak{e}_{7}^{\sigma_{3}}\right)^{\omega}=\mathfrak{s p}(4) .
$$

Further, $\omega$ acts on $\mathfrak{h}$ as $s_{\alpha_{2}} s_{\alpha_{5}} s_{\alpha_{7}}$, where $s_{\alpha}$ in the Weyl group is the reflection corresponding to the root $\alpha$.

5C. Types $\mathbf{E}_{\mathbf{8}}, \mathbf{F}_{\mathbf{4}}, \mathbf{G}_{\mathbf{2}}$. If $\mathfrak{u}_{0}=\mathfrak{e}_{8}, \mathfrak{f}_{4}, \mathfrak{g}_{2}$, then $\operatorname{Aut}\left(\mathfrak{u}_{0}\right)$ is connected and simply connected. By Proposition 4.1, Aut $\left(\mathfrak{u}_{0}\right)^{\theta}$ is connected. Then they are determined by $\mathfrak{u}_{0}^{\theta}$ and $\mathfrak{p}=\mathfrak{g}^{-\theta}$.

\section{Klein four-subgroups of $\operatorname{Aut}\left(\mathfrak{u}_{0}\right)$}

In this section, we classify Klein four-subgroups $\Gamma$ (called simply Klein subgroups) in $\operatorname{Aut}\left(\mathfrak{u}_{0}\right)$ up to conjugation. We also determine the fixed-point subgroups $\operatorname{Aut}\left(\mathfrak{u}_{0}\right)^{\Gamma}$. Note that such a $\Gamma$ is equal to $\{1, \theta, \sigma, \theta \sigma\}$ for two commuting involutions $\theta \neq \sigma$. Fix an involution $\theta$; the conjugacy class of $\Gamma$ is determined by the conjugacy classes of the involution $\sigma(\neq \theta)$ in $\operatorname{Aut}\left(\mathfrak{u}_{0}\right)^{\theta}$.

\section{A. Ordered commuting pairs of involutions and semisimple symmetric pairs.}

For a compact simple Lie algebra $\mathfrak{u}_{0}$ and its complexification $\mathfrak{g}$, the isomorphism classes of semisimple symmetric pairs $\left(\mathfrak{g}_{0}, \mathfrak{h}_{0}\right)$ with $\mathfrak{g}_{0}$ a real form of $\mathfrak{g}$ and $\mathfrak{h}_{0}\left(\neq \mathfrak{g}_{0}\right)$ noncompact are in one-to-one correspondence with the conjugacy classes of ordered commuting pairs of involutions $(\theta, \sigma)$ in $\operatorname{Aut}\left(\mathfrak{u}_{0}\right)$ with $\theta \neq \sigma$. One direction of this correspondence is as follows: let $\mathfrak{u}_{i, j}(i, j=0$ or 1$)$ be the joint eigenspace of $\theta$ and $\sigma$ where $\theta$ acts on it as $(-1)^{i}$ and $\sigma$ acts on it as $(-1)^{j}$. Then we have a decomposition

$$
\mathfrak{u}_{0}=\mathfrak{u}_{0,0} \oplus \mathfrak{u}_{0,1} \oplus \mathfrak{u}_{1,0} \oplus \mathfrak{u}_{1,1}
$$

Then $\mathfrak{k}_{0}=\mathfrak{u}_{0}^{\theta}=\mathfrak{u}_{0,0} \oplus \mathfrak{u}_{0,1}$ and $i \mathfrak{p}_{0}=\mathfrak{u}_{0}^{-\theta}=\mathfrak{u}_{1,0} \oplus \mathfrak{u}_{1,1}$. Extend $\theta, \sigma$ to holomorphic automorphisms of $\mathfrak{g}$ and let

$$
\mathfrak{g}_{0}=\mathfrak{k}_{0}+\mathfrak{p}_{0}=\mathfrak{u}_{0,0}+\mathfrak{u}_{0,1}+i\left(\mathfrak{u}_{1,0}+\mathfrak{u}_{1,1}\right) \quad \text { and } \quad \mathfrak{h}_{0}=\mathfrak{g}_{0}^{\sigma}=\mathfrak{u}_{0,0}+i \mathfrak{u}_{1,0} .
$$




\begin{tabular}{|cccc|}
\hline $\mathfrak{u}_{0}$ & $\Gamma_{i}$ & $\mathfrak{l}_{0}=\mathfrak{u}_{0} \Gamma_{i}$ & Type \\
\hline $\mathfrak{s u}(p+q)$ & $\Gamma_{p, q}=\left\langle\tau, I_{p, q}\right\rangle$ & $\mathfrak{s o}(p)+\mathfrak{s o}(q)$ & AI-AI-AIII, $\mathrm{S}$ \\
$\mathfrak{s u}(2 p)$ & $\Gamma_{p}=\left\langle\tau, J_{p}\right\rangle$ & $\mathfrak{u}(p)$ & AI-AII-AIII, N \\
$\mathfrak{s u}(2 p+2 q)$ & $\Gamma_{p, q}^{\prime}=\left\langle\tau J_{p+q}, I_{p, q}^{\prime}\right\rangle$ & $\mathfrak{s p}(p)+\mathfrak{s p}(q)$ & AII-AII-AII, $\mathrm{S}$ \\
$\mathfrak{s u}(p+q+r+s)$ & $\Gamma_{p, q, r, s}$ & $\mathfrak{s}(\mathfrak{u}(p)+\mathfrak{u}(q)+\mathfrak{u}(r)+\mathfrak{u}(s))$ & AIII-AIII-AIII, NSV \\
$\mathfrak{s u}(2 p)$ & $\Gamma_{p}=\left\langle I_{p, p}, J_{p}\right\rangle$ & $\mathfrak{s u}(p)$ & AIII-AIII-AIII, V \\
$\mathfrak{s o}(p+q+r+s)$ & $\Gamma_{p, q, r, s}$ & $\mathfrak{s o}(p)+\mathfrak{s o}(q)+\mathfrak{s o}(r)+\mathfrak{s o}(s))$ & BDI-BDI-BDI, NSV \\
$\mathfrak{s o}(2 p)$ & $\Gamma_{p}=\left\langle J_{p}, I_{p, p}\right\rangle$ & $\mathfrak{s o}(p)$ & DI-DI-DIII, $\mathrm{S}$ \\
$\mathfrak{s o}(2 p+2 q)$ & $\Gamma_{p, q}=\left\langle J_{p+q}, I_{p, q}^{\prime}\right\rangle$ & $\mathfrak{u}(p)+\mathfrak{u}(q)$ & DI-DIII-DIII, $\mathrm{S}$ \\
$\mathfrak{s o}(4 p)$ & $\Gamma_{p}^{\prime}=\left\langle J_{2 p}, K_{p}\right\rangle$ & $\mathfrak{s p}(p)$ & DIII-DIII-DIII, V \\
$\mathfrak{s p}(p)$ & $\Gamma_{p}=\langle\mathbf{i} I, \mathbf{j} I\rangle$ & $\mathfrak{s o}(p)$ & CI-CI-CI, V \\
$\mathfrak{s p}(p+q)$ & $\Gamma_{p, q}=\left\langle\mathbf{i} I, I_{p, q}\right\rangle$ & $\mathfrak{u}(p)+\mathfrak{u}(q)$ & CI-CI-CII, $\mathrm{S}$ \\
$\mathfrak{s p}(2 p)$ & $\Gamma_{p}^{\prime}=\left\langle\mathbf{i} I, \mathbf{j} J_{p}\right\rangle$ & $\mathfrak{s p}(p)$ & CI-CII-CII,, \\
$\mathfrak{s p}(p+q+r+s)$ & $\Gamma_{p, q, r, s}$ & $\mathfrak{s p}(p)+\mathfrak{s p}(q)+\mathfrak{s p}(r)+\mathfrak{s p}(s)$ & CII-CII-CII, NSV \\
\hline
\end{tabular}

Table 3. Klein subgroups in $\operatorname{Aut}\left(\mathfrak{u}_{0}\right)$ for the classical cases. (When $p=1, q=3, \Gamma_{1,3}$ is very special since $\operatorname{Ad}\left(I_{2,6}\right) \sim \operatorname{Ad}\left(J_{4}\right)$.)

Then $\mathfrak{g}_{0}$ is a real form of $\mathfrak{g}$ and $\left(\mathfrak{g}_{0}, \mathfrak{h}_{0}\right)$ is a semisimple symmetric pair with $\mathfrak{h}_{0} \neq \mathfrak{g}_{0}$ and noncompact. The other direction of this correspondence needs a more sophisticated argument.

When $\theta$ is fixed, the conjugacy classes of the pairs $(\theta, \sigma)$ in $\operatorname{Aut}\left(\mathfrak{u}_{0}\right)$ are in one-to-one correspondence with the $\operatorname{Aut}\left(\mathfrak{u}_{0}\right)^{\theta}$-conjugacy classes of involutions in $\operatorname{Aut}\left(\mathfrak{u}_{0}\right)^{\theta}-\{\theta\}$.

For an exceptional compact simple Lie algebra $\mathfrak{u}_{0}$ and any representative $\theta$ of involution classes in Section 3, we give the representatives of classes of involutions in $\operatorname{Aut}\left(\mathfrak{u}_{0}\right)^{\theta}-\{\theta\}$ and identify their classes in $\operatorname{Aut}\left(\mathfrak{u}_{0}\right)$. For any classical compact simple Lie algebra $\mathfrak{u}_{0}$ and a representative $\theta$ of an involution class, we have a similar classification of involutions in $\operatorname{Aut}\left(\mathfrak{u}_{0}\right)^{\theta}-\{\theta\}$; we omit it here but remark that the representatives can be constructed from Table 3. This gives a new proof to Berger's classification of semisimple symmetric pairs.

In most cases the symmetric subgroup $\operatorname{Aut}\left(\mathfrak{u}_{0}\right)^{\theta}$ is a product of classical groups with some twisting, for which we can classify their involution classes by matrix calculations. In the remaining cases, $\mathfrak{u}_{0}^{\theta}=\mathfrak{s}_{0} \oplus \mathfrak{z}$ for an exceptional simple Lie algebra $\mathfrak{s}_{0}$ and an algebra $\mathfrak{z}=0, i \mathbb{R}$ or $\mathfrak{s p}(1)$. We have a homomorphism

$$
p: \operatorname{Aut}\left(\mathfrak{u}_{0}\right)^{\theta} \rightarrow \operatorname{Aut}\left(\mathfrak{s}_{0}\right) .
$$

Then what we need to do is to classify involutions in $p^{-1}(\sigma)$ for $\sigma \in \operatorname{Aut}\left(\mathfrak{s}_{0}\right)$ an involution or the identity element, which is not hard in general.

For an exceptional compact simple Lie algebra $\mathfrak{u}_{0}$, the conjugacy class of an involution $\sigma \in \operatorname{Aut}\left(\mathfrak{u}_{0}\right)$ is determined by $\operatorname{dim} \mathfrak{g}^{\sigma}$. (This is an accidental phenomenon 
observed by Helgason [2001, pp. 517-518].) For any involution $\sigma \in \operatorname{Aut}\left(\mathfrak{u}_{0}\right)^{\theta}-\{\theta\}$, the class of $\sigma$ in $\operatorname{Aut}\left(\mathfrak{u}_{0}\right)$ is determined by $\operatorname{dim} \mathfrak{g}^{\sigma}=\operatorname{dim} \mathfrak{k}^{\sigma}+\operatorname{dim} \mathfrak{p}^{\sigma}$ and the dimensions $\operatorname{dim} \mathfrak{k}^{\sigma}, \operatorname{dim} \mathfrak{p}^{\sigma}$ can be calculated from the class of $\sigma$ in $\operatorname{Aut}\left(\mathfrak{u}_{0}\right)^{\theta}$. The coroots $H_{i}^{\prime}$ are defined in (2) and (5) and the involutions $\sigma_{i}, \sigma, \tau$ are defined in Section 3.

Type $\mathbf{E}_{6}$. Now $\mathfrak{u}_{0}=\mathfrak{e}_{6}$. For $\theta=\sigma_{1}=\exp \left(\pi i H_{2}^{\prime}\right)$, one has

$$
\operatorname{Aut}\left(\mathfrak{u}_{0}\right)^{\sigma_{1}}=\left(\operatorname{SU}(6) \times \operatorname{Sp}(1) /\left\langle\left(e^{2 \pi i / 3} I, 1\right),(-I,-1)\right\rangle\right) \rtimes\langle\tau\rangle,
$$

$\sigma_{1}=(I,-1)=(-I, 1)$, where $\operatorname{Ad}(\tau)(X, Y)=\left(J_{3} \bar{X} J_{3}^{-1}, Y\right)$. Then, in $\operatorname{Aut}\left(\mathfrak{u}_{0}\right)$,

$$
\begin{gathered}
\left(\left(\begin{array}{cc}
-I_{4} & 0 \\
0 & I_{2}
\end{array}\right), 1\right) \sim \sigma_{2}, \quad\left(\left(\begin{array}{cc}
-I_{2} & 0 \\
0 & I_{4}
\end{array}\right), 1\right) \sim \sigma_{1}, \\
\left(\left(\begin{array}{cc}
i I_{5} & 0 \\
0 & -i I_{1}
\end{array}\right), \mathbf{i}\right) \sim \sigma_{2}, \quad\left(\left(\begin{array}{cc}
i I_{3} & 0 \\
0 & -i I_{3}
\end{array}\right), \mathbf{i}\right) \sim \sigma_{1}, \\
\tau \sim \sigma_{3}, \quad \tau \sigma_{1} \sim \sigma_{4}, \quad \tau\left(J_{3}, \mathbf{i}\right) \sim \sigma_{4} .
\end{gathered}
$$

These elements represent all the conjugacy classes of involutions in $\operatorname{Aut}\left(\mathfrak{u}_{0}\right)^{\theta}-\{\theta\}$.

For $\theta=\sigma_{2}=\exp \left(\pi i\left(H_{1}^{\prime}+H_{6}^{\prime}\right)\right)$, one has

$$
\operatorname{Aut}\left(\mathfrak{u}_{0}\right)^{\sigma_{2}}=((\operatorname{Spin}(10) \times \mathrm{U}(1)) /\langle(c, i)\rangle) \rtimes\langle\tau\rangle, \quad \sigma_{2}=(-1,1)=(1,-1),
$$

where $c=e_{1} e_{2} \cdots e_{10}$ and $\operatorname{Ad}(\tau)(x, z)=\left(\left(e_{1} e_{2} \cdots e_{9}\right) x\left(e_{1} e_{2} \cdots e_{9}\right)^{-1}, z^{-1}\right)$. Then, in $\operatorname{Aut}\left(\mathfrak{u}_{0}\right)$,

$$
\begin{gathered}
\left(e_{1} e_{2} e_{3} e_{4}, 1\right) \sim \sigma_{1}, \quad\left(e_{1} e_{2} \cdots e_{8}, 1\right) \sim \sigma_{2}, \\
\left(\delta, \frac{1+i}{\sqrt{2}}\right) \sim \sigma_{2}, \quad\left(-\delta, \frac{1+i}{\sqrt{2}}\right) \sim \sigma_{1}, \\
\tau \sim \sigma_{3}, \quad \tau\left(e_{1} e_{2} e_{3} e_{4}, 1\right) \sim \sigma_{4}
\end{gathered}
$$

where

$$
\delta=\frac{1+e_{1} e_{2}}{\sqrt{2}} \frac{1+e_{3} e_{4}}{\sqrt{2}} \cdots \frac{1+e_{9} e_{10}}{\sqrt{2}} .
$$

These elements represent all the conjugacy classes of involutions in $\operatorname{Aut}\left(\mathfrak{u}_{0}\right)^{\theta}-\{\theta\}$. For $\theta=\sigma_{3}=\tau$, one has $\operatorname{Aut}\left(\mathfrak{u}_{0}\right)^{\sigma_{3}}=\mathrm{F}_{4} \times\langle\tau\rangle$. Let $\tau_{1}, \tau_{2}$ be involutions in $\mathrm{F}_{4}$ with

$$
\mathfrak{f}_{4}^{\tau_{1}} \cong \mathfrak{s p}(3) \oplus \mathfrak{s p}(1), \quad \mathfrak{f}_{4}^{\tau_{2}} \cong \mathfrak{s o}(9)
$$

Then, in $\operatorname{Aut}\left(\mathfrak{u}_{0}\right)$,

$$
\begin{aligned}
& \tau_{1} \sim \sigma_{1}, \quad \tau_{2} \sim \sigma_{2}, \\
& \sigma_{3} \tau_{1} \sim \sigma_{4}, \quad \\
& \sigma_{3} \tau_{2} \sim \sigma_{3},
\end{aligned}
$$

these elements represent all the conjugacy classes of involutions in $\operatorname{Aut}\left(\mathfrak{u}_{0}\right)^{\theta}-\{\theta\}$. 
For $\theta=\sigma_{4}=\tau \exp \left(\pi i H_{2}^{\prime}\right)$, one has $\operatorname{Aut}\left(\mathfrak{u}_{0}\right)^{\sigma_{4}}=(\operatorname{Sp}(4) /\langle-I\rangle) \times\left\langle\sigma_{4}\right\rangle$. Let

$$
\tau_{1}=\mathbf{i} I, \quad \tau_{2}=\left(\begin{array}{cc}
-I_{2} & 0 \\
0 & I_{2}
\end{array}\right), \quad \tau_{3}=\left(\begin{array}{cc}
-1 & 0 \\
0 & I_{3}
\end{array}\right) .
$$

Then, in $\operatorname{Aut}\left(\mathfrak{u}_{0}\right)$,

$$
\begin{array}{rlrl}
\tau_{1} & \sim \sigma_{1}, & \tau_{2} & \sim \sigma_{2}, \\
\sigma_{4} \tau_{1} & \sim \sigma_{4}, \quad \sigma_{3} & \sim \sigma_{1}, \\
\sigma_{2} & \sim \sigma_{4}, \quad \sigma_{4} \tau_{3} & \sim \sigma_{3} .
\end{array}
$$

These elements represent all the conjugacy classes of involutions in $\operatorname{Aut}\left(\mathfrak{u}_{0}\right)^{\theta}-\{\theta\}$. Type $\mathbf{E}_{7}$. Now $\mathfrak{u}_{0}=\mathfrak{e}_{7}$. For $\theta=\sigma_{1}=\exp \left(\pi i H_{2}^{\prime}\right)$, one has

$$
\operatorname{Aut}\left(\mathfrak{u}_{0}\right)^{\sigma_{1}}=(\operatorname{Spin}(12) \times \operatorname{Sp}(1)) /\langle(c, 1),(-1,-1)\rangle,
$$

where $\sigma_{1}=(-1,1)=(1,-1), c=e_{1} e_{2} \cdots e_{12}$. Let

$$
\delta=\frac{1+e_{1} e_{2}}{\sqrt{2}} \frac{1+e_{3} e_{4}}{\sqrt{2}} \cdots \frac{1+e_{11} e_{12}}{\sqrt{2}} .
$$

Then, in $\operatorname{Aut}\left(\mathfrak{u}_{0}\right)$,

$$
\begin{aligned}
\left(e_{1} e_{2} e_{3} e_{4}, 1\right) & \sim \sigma_{1}, \quad\left(e_{1} e_{2}, \mathbf{i}\right) \sim \sigma_{2}, & \left(e_{1} e_{2} \cdots e_{6}, \mathbf{i}\right) & \sim \sigma_{3}, \\
(\delta, 1) & \sim \sigma_{2}, \quad(-\delta, 1) \sim \sigma_{3}, & \left(e_{1} \delta e_{1}, \mathbf{i}\right) & \sim \sigma_{1} .
\end{aligned}
$$

These elements represent all conjugacy classes of involutions in $\operatorname{Aut}\left(\mathfrak{u}_{0}\right)^{\theta}-\{\theta\}$. Moreover,

$$
\left\langle\sigma_{1},\left(e_{1} e_{2} e_{3} e_{4}, 1\right)\right\rangle \sim F_{2}, \quad\left\langle\sigma_{1},\left(e_{1} \delta e_{1}, \mathbf{i}\right)\right\rangle \sim F_{1} .
$$

For $\theta=\sigma_{2}=\tau=\exp \left(\pi i \frac{H_{2}^{\prime}+H_{5}^{\prime}+H_{7}^{\prime}}{2}\right)$, one has

$$
\operatorname{Aut}\left(\mathfrak{u}_{0}\right)_{0}^{\sigma_{2}}=\left(\left(\mathrm{E}_{6} \times \mathrm{U}(1)\right) /\left\langle\left(c, e^{\frac{2 \pi i}{3}}\right)\right\rangle\right) \rtimes\langle\omega\rangle,
$$

where $c$ is a nontrivial central element of $\mathrm{E}_{6}$ with $o(c)=3, \sigma_{2}=(1,-1)$ and $\left(\mathfrak{e}_{6} \oplus i \mathbb{R}\right)^{\omega}=\mathfrak{f}_{4} \oplus 0$. Let $\tau_{1}, \tau_{2}$ be involutions in $\mathrm{E}_{6}$ with

$$
\mathfrak{e}_{6}^{\tau_{1}} \cong \mathfrak{s u}(6) \oplus \mathfrak{s p}(1), \quad \mathfrak{e}_{6}^{\tau_{2}} \cong \mathfrak{s o}(10) \oplus i \mathbb{R} .
$$

Then, in $\operatorname{Aut}\left(\mathfrak{u}_{0}\right)$,

$$
\begin{array}{rlrl}
\tau_{1} & \sim \sigma_{1}, & \tau_{2} & \sim \sigma_{1}, \\
\tau_{1} \sigma_{2} & \sim \sigma_{3}, & \tau_{2} \sigma_{2} & \sim \sigma_{2}, \\
\omega & \sim \sigma_{2}, \quad \omega \eta & \sim \sigma_{3},
\end{array}
$$

where $\eta \in \mathrm{F}_{4}=\mathrm{E}_{6}^{\omega}$ is an involution with $\left(\mathfrak{f}_{4}\right)^{\eta} \cong \mathfrak{s p}(3) \oplus \mathfrak{s p}(1)$. These elements represent all the conjugacy classes of involutions in $\operatorname{Aut}\left(\mathfrak{u}_{0}\right)^{\theta}-\{\theta\}$. 
For

one has

$$
\theta=\sigma_{3}=\exp \left(\pi i \frac{H_{2}^{\prime}+H_{5}^{\prime}+H_{7}^{\prime}+2 H_{1}^{\prime}}{2}\right)
$$

$$
\operatorname{Aut}\left(\mathfrak{u}_{0}\right)_{0}^{\sigma_{3}}=(\mathrm{SU}(8) /\langle i I\rangle) \rtimes\langle\omega\rangle, \quad \sigma_{3}=\frac{1+i}{\sqrt{2}} I,
$$

where $\operatorname{Ad}(\omega) X=J_{4} \bar{X} J_{4}^{-1}$. Let $\tau_{1}=\left(\begin{array}{cc}{ }^{-I_{2}} & \\ & I_{6}\end{array}\right), \tau_{2}=\left(\begin{array}{ll}{ }^{-I_{4}} & \\ & I_{4}\end{array}\right)$. Then, in $\operatorname{Aut}\left(\mathfrak{u}_{0}\right)$,

$$
\begin{gathered}
\tau_{1} \sim \sigma_{1}, \quad \tau_{2} \sim \sigma_{1}, \quad \tau_{1} \sigma_{3} \sim \sigma_{2}, \quad \tau_{2} \sigma_{3} \sim \sigma_{3}, \\
\omega \sim \sigma_{2}, \quad \omega \sigma_{3} \sim \sigma_{3}, \quad \omega J_{4} \sim \sigma_{3} .
\end{gathered}
$$

These elements represent all conjugacy classes of involutions in $\operatorname{Aut}\left(\mathfrak{u}_{0}\right)^{\theta}-\{\theta\}$. Type $\mathbf{E}_{\mathbf{8}}$. Now $\mathfrak{u}_{0}=\mathfrak{e}_{8}$. For $\theta=\sigma_{1}=\exp \left(\pi i H_{2}^{\prime}\right)$, one has

$$
\operatorname{Aut}\left(\mathfrak{u}_{0}\right)^{\sigma_{1}} \cong\left(\mathrm{E}_{7} \times \operatorname{Sp}(1)\right) /\langle(c,-1)\rangle,
$$

where $\sigma_{1}=(1,-1)=(c, 1)$. Let $\tau_{1}, \tau_{2}$ denote the elements in $\mathrm{E}_{7}$ with $\tau_{1}^{2}=\tau_{2}^{2}=c$ and $\mathfrak{e}_{7}^{\tau_{1}} \cong \mathfrak{e}_{6} \oplus i \mathbb{R}, \mathfrak{e}_{7}^{\tau_{2}} \cong \mathfrak{s u}(8)$. Let $\tau_{3}, \tau_{4}$ be involutions in $\mathrm{E}_{7}$ such that there exist Klein subgroups $\Gamma, \Gamma^{\prime} \subset E_{7}$ with three nonidentity elements in $\Gamma$ all conjugate to $\tau_{3}$, three nonidentity elements in $\Gamma^{\prime}$ all conjugate to $\tau_{4}$, and $\mathfrak{e}_{7}^{\Gamma} \cong \mathfrak{s u}(6) \oplus(i \mathbb{R})^{2}$, $\mathfrak{e}_{7}^{\Gamma^{\prime}} \cong \mathfrak{s o}(8) \oplus(\mathfrak{s p}(1))^{3}$. Then, in $\operatorname{Aut}\left(\mathfrak{u}_{0}\right)$,

$$
\left(\tau_{1}, \mathbf{i}\right) \sim \sigma_{1}, \quad\left(\tau_{2}, \mathbf{i}\right) \sim \sigma_{2}, \quad\left(\tau_{3}, 1\right) \sim \sigma_{1}, \quad\left(\tau_{4}, 1\right) \sim \sigma_{2} .
$$

These elements represent all conjugacy classes of involutions in $\operatorname{Aut}\left(\mathfrak{u}_{0}\right)^{\theta}-\{\theta\}$.

For $\theta=\sigma_{2}=\exp \left(\pi i\left(H_{2}^{\prime}+H_{1}^{\prime}\right)\right)$, one has $\operatorname{Aut}\left(\mathfrak{u}_{0}\right)^{\sigma_{2}} \cong \operatorname{Spin}(16) /\langle c\rangle$, where $\sigma_{2}=-1, c=e_{1} e_{2} \cdots e_{16}$. Let

$$
\begin{gathered}
\delta=\frac{1+e_{1} e_{2}}{\sqrt{2}} \frac{1+e_{3} e_{4}}{\sqrt{2}} \cdots \frac{1+e_{15} e_{16}}{\sqrt{2}}, \\
\tau_{1}=e_{1} e_{2} e_{3} e_{4}, \quad \tau_{2}=e_{1} e_{2} e_{3} \cdots e_{8}, \quad \tau_{3}=\delta, \quad \tau_{4}=-\delta .
\end{gathered}
$$

Then, in $\operatorname{Aut}\left(\mathfrak{u}_{0}\right)$,

$$
\tau_{1} \sim \sigma_{1}, \quad \tau_{2} \sim \sigma_{2}, \quad \tau_{3} \sim \sigma_{1}, \quad \tau_{4} \sim \sigma_{2} .
$$

These elements represent all the conjugacy classes of involutions in $\operatorname{Aut}\left(\mathfrak{u}_{0}\right)^{\theta}-\{\theta\}$. Type $\mathbf{F}_{4}$. When $\mathfrak{u}_{0}=\mathfrak{f}_{4}$, for $\theta=\sigma_{1}=\exp \left(\pi i H_{1}^{\prime}\right)$,

$$
\operatorname{Aut}\left(\mathfrak{u}_{0}\right)^{\sigma_{1}} \cong \operatorname{Sp}(3) \times \operatorname{Sp}(1) /\langle(-I,-1)\rangle,
$$

where $\sigma_{1}=(-I, 1)=(I,-1)$. Let

$$
\tau_{1}=\left(\left(\begin{array}{ccc}
-1 & 0 & 0 \\
0 & 1 & 0 \\
0 & 0 & 1
\end{array}\right), 1\right), \quad \tau_{2}=\left(\left(\begin{array}{ccc}
-1 & 0 & 0 \\
0 & -1 & 0 \\
0 & 0 & 1
\end{array}\right), 1\right), \quad \tau_{3}=(\mathbf{i} I, \mathbf{i}) .
$$


Then, in $\operatorname{Aut}\left(\mathfrak{u}_{0}\right)$,

$$
\tau_{1} \sim \sigma_{1}, \quad \tau_{2} \sim \sigma_{2}, \quad \tau_{3} \sim \sigma_{1} .
$$

These elements represent all conjugacy classes of involutions in $\operatorname{Aut}\left(\mathfrak{u}_{0}\right)^{\theta}-\{\theta\}$.

For $\theta=\sigma_{2}=\exp \left(\pi i H_{4}^{\prime}\right)$, one has $\operatorname{Aut}\left(\mathfrak{u}_{0}\right)^{\sigma_{2}} \cong \operatorname{Spin}(9), \sigma_{2}=-1$. Let $\tau_{1}=$ $e_{1} e_{2} e_{3} e_{4}, \tau_{2}=e_{1} e_{2} e_{3} \cdots e_{8}$. Then, in Aut $\left(\mathfrak{u}_{0}\right)$, we have $\tau_{1} \sim \sigma_{1}$ and $\tau_{2} \sim \sigma_{2}$. These elements represent all conjugacy classes of involutions in $\operatorname{Aut}\left(\mathfrak{u}_{0}\right)^{\theta}-\{\theta\}$.

Type $\mathbf{G}_{2}$. When $\mathfrak{u}_{0}=\mathfrak{g}_{2}$ and $\theta=\sigma=\exp \left(\pi i H_{1}^{\prime}\right)$, one has

$$
\operatorname{Aut}\left(\mathfrak{u}_{0}\right)^{\sigma_{1}} \cong \operatorname{Sp}(1) \times \operatorname{Sp}(1) /\langle(-1,-1)\rangle,
$$

where $\sigma_{1}=(-1,1)=(1,-1)$. Denote $\tau=(\mathbf{i}, \mathbf{i})$. Then, in Aut $\left(\mathfrak{u}_{0}\right)$, we have $\tau \sim \sigma$, and $\tau$ represents the unique conjugacy class of involutions in $\operatorname{Aut}\left(\mathfrak{u}_{0}\right)^{\theta}-\{\theta\}$.

By the above, we have reproved Berger's classification of semisimple symmetric pairs. The next proposition is an immediate consequence of this classification.

Proposition 6.1. There are 23, 19, 8, 5, and 1 isomorphism classes of nontrivial (that is, $\left.\mathfrak{h}_{0} \neq \mathfrak{g}_{0}\right)$ semisimple symmetric pairs $\left(\mathfrak{g}_{0}, \mathfrak{h}_{0}\right)$ with $\mathfrak{g}_{0}$ noncompact and $\mathfrak{g}=\mathfrak{g}_{0} \otimes_{\mathbb{R}} \mathbb{C}$ a complex simple Lie algebra of types $\mathbf{E}_{\mathbf{6}}, \mathbf{E}_{\mathbf{7}}, \mathbf{E}_{\mathbf{8}}, \mathbf{F}_{\mathbf{4}}$, and $\mathbf{G}_{\mathbf{2}}$, respectively.

6B. Klein subgroups, speciality, regularity and centralizers. For a Klein group $\Gamma \subset \operatorname{Aut}\left(\mathfrak{u}_{0}\right)$, we call the conjugacy classes of the involutions in $\Gamma$ the involution type of $\Gamma$, and the classes of Riemannian symmetric pairs corresponding to the involutions in $\Gamma$ the symmetric space type of $\Gamma$. Since there is a one-to-one correspondence between these two types, we simply say type of $\Gamma$ for either involution type or symmetric space type.

For a compact simple Lie algebra $\mathfrak{u}_{0}$, a Klein subgroup $\Gamma$ of $\operatorname{Aut}\left(\mathfrak{u}_{0}\right)$ is called regular if any two distinct conjugate (in $\operatorname{Aut}\left(\mathfrak{u}_{0}\right)$ ) elements $\sigma, \theta \in \Gamma$ are conjugate by an element $g \in \operatorname{Aut}\left(\mathfrak{u}_{0}\right)$ commuting with $\theta \sigma$ (that is, $\left.g \in \operatorname{Aut}\left(\mathfrak{u}_{0}\right)^{\theta \sigma}\right)$.

A Klein subgroup $\Gamma \subset \operatorname{Aut}\left(\mathfrak{u}_{0}\right)$ is called special if there are two (distinct) elements of $\Gamma$ which are conjugate in $\operatorname{Aut}\left(\mathfrak{u}_{0}\right)$. It is called very special if three involutions of $\Gamma$ are pairwise conjugate in $\operatorname{Aut}\left(\mathfrak{u}_{0}\right)$. Otherwise it is called nonspecial. The definition of special is due to [Ōshima and Sekiguchi 1984].

In Tables 3 and 4 , we list some Klein subgroups $\Gamma_{i} \subset \operatorname{Aut}\left(\mathfrak{u}_{0}\right)$ for each compact simple Lie algebra $\mathfrak{u}_{0}$ together with their symmetric space types (when $\mathfrak{u}_{0}$ is classical) or involution types (when $\mathfrak{u}_{0}$ is exceptional). These subgroups are not conjugate to each other since their fixed point subalgebras $\mathfrak{u}_{0}^{\Gamma_{i}}$ are nonisomorphic. In the last column we also indicate whether they are special or not. For brevity, we write $\mathrm{N}$ to mean nonspecial, $\mathrm{S}$ to mean special but not very special, $\mathrm{V}$ to mean very special. The speciality of the subgroups $\Gamma_{p, q, r, s}$ depends on the parameters. In general they can be nonspecial, special or very special; in this case we use NSV to denote their 


\begin{tabular}{|c|c|c|c|}
\hline $\mathfrak{u}_{0}$ & $\Gamma_{i}$ & $\mathfrak{l}_{0}=\mathfrak{u}_{0}^{\Gamma_{i}}$ & Type \\
\hline $\mathfrak{e}_{6}$ & $\Gamma_{1}=\left\langle\exp \left(\pi i H_{2}^{\prime}\right), \exp \left(\pi i H_{4}^{\prime}\right)\right\rangle$ & $(\mathfrak{s u}(3))^{2} \oplus(i \mathbb{R})^{2}$ & $\left(\sigma_{1}, \sigma_{1}, \sigma_{1}\right), \mathrm{V}$ \\
\hline $\mathfrak{e}_{6}$ & $\Gamma_{2}=\left\langle\exp \left(\pi i H_{4}^{\prime}\right), \exp \left(\pi i\left(H_{3}^{\prime}+H_{4}^{\prime}+H_{5}^{\prime}\right)\right)\right\rangle$ & $\mathfrak{s u}(4) \oplus(\mathfrak{s p}(1))^{2} \oplus i \mathbb{R}$ & $\left(\sigma_{1}, \sigma_{1}, \sigma_{2}\right), \mathrm{S}$ \\
\hline $\mathfrak{e}_{6}$ & $\Gamma_{3}=\left\langle\exp \left(\pi i\left(H_{2}^{\prime}+H_{1}^{\prime}\right)\right), \exp \left(\pi i\left(H_{4}^{\prime}+H_{1}^{\prime}\right)\right)\right\rangle$ & $\mathfrak{s u}(5) \oplus(i \mathbb{R})^{2}$ & $\left(\sigma_{1}, \sigma_{2}, \sigma_{2}\right), \mathrm{S}$ \\
\hline $\mathfrak{e}_{6}$ & $\Gamma_{4}=\left\langle\exp \left(\pi i\left(H_{1}^{\prime}+H_{6}^{\prime}\right)\right), \exp \left(\pi i\left(H_{3}^{\prime}+H_{5}^{\prime}\right)\right)\right\rangle$ & $\mathfrak{s o}(8) \oplus(i \mathbb{R})^{2}$ & $\left(\sigma_{2}, \sigma_{2}, \sigma_{2}\right), \mathrm{V}$ \\
\hline $\mathfrak{e}_{6}$ & $\Gamma_{5}=\left\langle\exp \left(\pi i H_{2}^{\prime}\right), \tau\right\rangle$ & $\mathfrak{s p}(3) \oplus \mathfrak{s p}(1)$ & $\left(\sigma_{1}, \sigma_{3}, \sigma_{4}\right), \mathrm{N}$ \\
\hline $\mathfrak{e}_{6}$ & $\Gamma_{6}=\left\langle\exp \left(\pi i H_{2}^{\prime}\right), \tau \exp \left(\pi i H_{4}^{\prime}\right)\right\rangle$ & $\mathfrak{s o}(6) \oplus i \mathbb{R}$ & $\left(\sigma_{1}, \sigma_{4}, \sigma_{4}\right), \mathrm{S}$ \\
\hline $\mathfrak{e}_{6}$ & $\left.\Gamma_{7}=\left\langle\exp \left(\pi i\left(H_{1}^{\prime}+H_{6}^{\prime}\right)\right)\right), \tau\right\rangle$ & $\mathfrak{s o}(9)$ & $\left(\sigma_{2}, \sigma_{3}, \sigma_{3}\right), \mathrm{S}$ \\
\hline $\mathfrak{e}_{6}$ & $\Gamma_{8}=\left\langle\exp \left(\pi i\left(H_{1}^{\prime}+H_{6}^{\prime}\right)\right), \tau \exp \left(\pi i H_{2}^{\prime}\right)\right\rangle$ & $\mathfrak{s o}(5) \oplus \mathfrak{s o}(5)$ & $\left(\sigma_{2}, \sigma_{4}, \sigma_{4}\right), \mathrm{S}$ \\
\hline $\mathfrak{e}_{7}$ & $\Gamma_{1}=\left\langle\exp \left(\pi i H_{2}^{\prime}\right), \exp \left(\pi i H_{4}^{\prime}\right)\right\rangle$ & $\mathfrak{s u}(6) \oplus(i \mathbb{R})^{2}$ & $\left(\sigma_{1}, \sigma_{1}, \sigma_{1}\right), \mathrm{V}$ \\
\hline $\mathfrak{e}_{7}$ & $\Gamma_{2}=\left\langle\exp \left(\pi i H_{2}^{\prime}\right), \exp \left(\pi i H_{3}^{\prime}\right)\right\rangle$ & $\mathfrak{s o}(8) \oplus(\mathfrak{s p}(1))^{3}$ & $\left(\sigma_{1}, \sigma_{1}, \sigma_{1}\right), \mathrm{V}$ \\
\hline $\mathfrak{e}_{7}$ & $\Gamma_{3}=\left\langle\exp \left(\pi i H_{2}^{\prime}\right), \tau\right\rangle$ & $\mathfrak{s o}(10) \oplus(i \mathbb{R})^{2}$ & $\left(\sigma_{1}, \sigma_{2}, \sigma_{2}\right), \mathrm{S}$ \\
\hline $\mathfrak{e}_{7}$ & $\Gamma_{4}=\left\langle\exp \left(\pi i H_{1}^{\prime}\right), \tau\right\rangle$ & $\mathfrak{s u}(6) \oplus \mathfrak{s p}(1) \oplus i \mathbb{R}$ & $\left(\sigma_{1}, \sigma_{2}, \sigma_{3}\right), \mathrm{N}$ \\
\hline $\mathfrak{e}_{7}$ & $\Gamma_{5}=\left\langle\exp \left(\pi i H_{2}^{\prime}\right), \tau \exp \left(\pi i H_{1}^{\prime}\right)\right\rangle$ & $\mathfrak{s u}(4) \oplus \mathfrak{s u}(4) \oplus i \mathbb{R}$ & $\left(\sigma_{1}, \sigma_{3}, \sigma_{3}\right), \mathrm{S}$ \\
\hline $\mathfrak{e}_{7}$ & $\Gamma_{6}=\langle\tau, \omega\rangle$ & $\mathfrak{f}_{4}$ & $\left(\sigma_{2}, \sigma_{2}, \sigma_{2}\right), \mathrm{V}$ \\
\hline $\mathfrak{e}_{7}$ & $\Gamma_{7}=\left\langle\tau, \omega \exp \left(\pi i H_{1}^{\prime}\right)\right\rangle$ & $\mathfrak{s p}(4)$ & $\left(\sigma_{2}, \sigma_{3}, \sigma_{3}\right), \mathrm{S}$ \\
\hline $\mathfrak{e}_{7}$ & $\Gamma_{8}=\left\langle\tau \exp \left(\pi i H_{1}^{\prime}\right), \omega \exp \left(\pi i H_{3}^{\prime}\right)\right\rangle$ & $\mathfrak{s o}(8)$ & $\left(\sigma_{3}, \sigma_{3}, \sigma_{3}\right), \mathrm{V}$ \\
\hline $\mathfrak{e}_{8}$ & $\Gamma_{1}=\left\langle\exp \left(\pi i H_{2}^{\prime}\right), \exp \left(\pi i H_{4}^{\prime}\right)\right\rangle$ & $\mathfrak{e}_{6} \oplus(i \mathbb{R})^{2}$ & $\left(\sigma_{1}, \sigma_{1}, \sigma_{1}\right), \mathrm{V}$ \\
\hline $\mathfrak{e}_{8}$ & $\Gamma_{2}=\left\langle\exp \left(\pi i H_{2}^{\prime}\right), \exp \left(\pi i H_{1}^{\prime}\right)\right\rangle$ & $\mathfrak{s o}(12) \oplus(\mathfrak{s p}(1))^{2}$ & $\left(\sigma_{1}, \sigma_{1}, \sigma_{2}\right), \mathrm{S}$ \\
\hline $\mathfrak{e}_{8}$ & $\Gamma_{3}=\left\langle\exp \left(\pi i H_{2}^{\prime}\right), \exp \left(\pi i\left(H_{1}^{\prime}+H_{4}^{\prime}\right)\right)\right\rangle$ & $\mathfrak{s u}(8) \oplus i \mathbb{R}$ & $\left(\sigma_{1}, \sigma_{2}, \sigma_{2}\right), \mathrm{S}$ \\
\hline $\mathfrak{e}_{8}$ & $\Gamma_{4}=\left\langle\exp \left(\pi i\left(H_{2}^{\prime}+H_{1}^{\prime}\right)\right), \exp \left(\pi i\left(H_{5}^{\prime}+H_{1}^{\prime}\right)\right)\right\rangle$ & $\mathfrak{s o}(8) \oplus \mathfrak{s o}(8)$ & $\left(\sigma_{2}, \sigma_{2}, \sigma_{2}\right), \mathrm{V}$ \\
\hline $\mathfrak{f}_{4}$ & $\Gamma_{1}=\left\langle\exp \left(\pi i H_{2}^{\prime}\right), \exp \left(\pi i H_{1}^{\prime}\right)\right\rangle$ & $\mathfrak{s u}(3) \oplus(i \mathbb{R})^{2}$ & $\left(\sigma_{1}, \sigma_{1}, \sigma_{1}\right), \mathrm{V}$ \\
\hline $\mathfrak{f}_{4}$ & $\Gamma_{2}=\left\langle\exp \left(\pi i H_{3}^{\prime}\right), \exp \left(\pi i H_{2}^{\prime}\right)\right\rangle$ & $\mathfrak{s o}(5) \oplus(\mathfrak{s p}(1))^{2}$ & $\left(\sigma_{1}, \sigma_{1}, \sigma_{2}\right), \mathrm{S}$ \\
\hline $\mathfrak{f}_{4}$ & $\Gamma_{3}=\left\langle\exp \left(\pi i H_{4}^{\prime}\right), \exp \left(\pi i H_{3}^{\prime}\right)\right\rangle$ & $\mathfrak{s o}(8)$ & $\left(\sigma_{2}, \sigma_{2}, \sigma_{2}\right), \mathrm{V}$ \\
\hline $\mathfrak{g}_{2}$ & $\Gamma=\left\langle\exp \left(\pi i H_{1}^{\prime}\right), \exp \left(\pi i H_{2}^{\prime}\right)\right\rangle$ & $(i \mathbb{R})^{2}$ & $(\sigma, \sigma, \sigma), \mathrm{V}$ \\
\hline
\end{tabular}

Table 4. Klein four-subgroups in $\operatorname{Aut}\left(\mathfrak{u}_{0}\right)$ for the exceptional cases.

speciality. The reader can determine for which parameters they are nonspecial, special or very special. The notation $I_{p, q}, J_{p}$, etc. is defined in Section $2 \mathrm{C}$.

Theorem 6.2. For a compact simple Lie algebra $\mathfrak{u}_{0}$, any Klein subgroup $\Gamma \subset \operatorname{Aut}\left(\mathfrak{u}_{0}\right)$ is conjugate to one in Table 3 or Table 4 and they are all regular.

Proof. When $\mathfrak{u}_{0}$ is a classical compact simple Lie algebra, we can do matrix calculation to show Table 3 is complete and any Klein subgroup is regular. When $\mathfrak{u}_{0}$ is an exceptional compact simple Lie algebra, from Klein subgroups we get nonconjugate commuting pairs of involutions $\left(\theta_{1}, \theta_{2}\right)$ distinguished by the isomorphism type of $\mathfrak{u}_{0}^{\left\langle\theta_{1}, \theta_{2}\right\rangle}$ or the distribution of the classes of the (ordered) tuples $\left(\theta_{1}, \theta_{2}, \theta_{3}\right)$. When $\mathfrak{u}_{0}$ is of type $\mathbf{E}_{\mathbf{6}}, \mathbf{E}_{\mathbf{7}}, \mathbf{E}_{\mathbf{8}}, \mathbf{F}_{\mathbf{4}}$, or $\mathbf{G}_{\mathbf{2}}$, we get (at least) $23,19,8,5$, or 1 nonconjugate commuting pairs, respectively. By Proposition 6.1, they represent all conjugacy classes of commuting pairs of involutions. So Table 4 is complete. 
For an exceptional simple Lie algebra $\mathfrak{u}_{0}$, suppose that some Klein subgroup fails to be regular. Then we can construct nonconjugate commuting pairs $\left(\theta_{1}, \theta_{2}\right)$ and $\left(\theta_{1}^{\prime}, \theta_{2}^{\prime}\right)\left(=\left(\theta_{2}, \theta_{1}\right)\right)$ with $\left\langle\theta_{1}, \theta_{2}\right\rangle=\left\langle\theta_{1}^{\prime}, \theta_{2}^{\prime}\right\rangle, \theta_{1} \sim \theta_{1}^{\prime}, \theta_{2} \sim \theta_{2}^{\prime}, \theta_{1} \theta_{2} \sim \theta_{1}^{\prime} \theta_{2}^{\prime}$. Then there should exist more isomorphism classes of semisimple symmetric pairs. But it is not the case, and it follows that any Klein subgroup is regular.

Another way of proving all Klein subgroups of $\operatorname{Aut}\left(\mathfrak{u}_{0}\right)$ are regular is as follows. First we just need to check for any commuting pair of involutions $\theta_{1}, \theta_{2} \in \operatorname{Aut}\left(\mathfrak{u}_{0}\right)$ with $\theta_{1} \sim \theta_{2}$ (in $\left.\operatorname{Aut}\left(\mathfrak{u}_{0}\right)\right), \theta_{1}, \theta_{2}$ are conjugate in $\operatorname{Aut}\left(\mathfrak{u}_{0}\right)^{\theta}$, where $\theta=\theta_{1} \theta_{2}$. Fix $\theta$ as a representative in Section 3, when $\mathfrak{u}_{0}$ is an exceptional simple Lie algebra. This was already checked in the last subsection; when $\mathfrak{u}_{0}$ is a classical simple Lie algebra, we can check this from the data in Table 3 (list of Klein groups with symmetric space type) and Table 2 (symmetric subgroups).

A statement equivalent to the regularity of all Klein subgroups (Theorem 6.2) is that two commuting pairs of involutions $(\theta, \sigma)$ and $\left(\theta^{\prime}, \sigma^{\prime}\right)$ are conjugate in $\operatorname{Aut}\left(\mathfrak{u}_{0}\right)$ if and only if

$$
\theta \sim \theta^{\prime}, \quad \sigma \sim \sigma^{\prime}, \quad \theta \sigma \sim \theta^{\prime} \sigma^{\prime}
$$

and the Klein subgroups $\langle\theta, \sigma\rangle,\left\langle\theta^{\prime}, \sigma^{\prime}\right\rangle$ are conjugate. This statement clearly implies the second statement in Theorem 6.2. To derive this statement from Theorem 6.2, give two pairs $(\theta, \sigma)$ and $\left(\theta^{\prime}, \sigma^{\prime}\right)$ with $\theta \sim \theta^{\prime}, \sigma \sim \sigma^{\prime}, \theta \sigma \sim \theta^{\prime} \sigma^{\prime}$ and $\langle\theta, \sigma\rangle \sim\left\langle\theta^{\prime}, \sigma^{\prime}\right\rangle$. After replacing $\left(\theta^{\prime}, \sigma^{\prime}\right)$ by a pair conjugate to it, we may assume $\langle\theta, \sigma\rangle=\left\langle\theta^{\prime}, \sigma^{\prime}\right\rangle$, that is, $(\theta, \sigma)$ and $\left(\theta^{\prime}, \sigma^{\prime}\right)$ generate the same Klein subgroup. By Theorem 6.2, $\langle\theta, \sigma\rangle$ is regular, so $(\theta, \sigma)$ and $\left(\theta^{\prime}, \sigma^{\prime}\right)$ are conjugate. Since any Klein subgroup of $\operatorname{Aut}\left(\mathfrak{u}_{0}\right)$ is regular, a conjugacy class of Klein subgroups gives 6, 3, or 1 isomorphism types of semisimple symmetric pairs when it is nonspecial, special but not very special, or very special, respectively.

The fact that all Klein subgroups in $\operatorname{Aut}\left(\mathfrak{u}_{0}\right)$ are regular is an interesting phenomenon. The property of regularity can be generalized to closed subgroups of any Lie group; a vast array of examples of nonregular subgroups is given in [Larsen 1994].

From Tables 1 and 4, we can abstract the following facts.

Proposition 6.3. When $\mathfrak{u}_{0}$ is an exceptional compact simple Lie algebra, any two classes of involutions have commuting representatives; for any Klein group $\Gamma \subset \operatorname{Aut}\left(\mathfrak{u}_{0}\right)$ the centralizer $\operatorname{Aut}\left(\mathfrak{u}_{0}\right)^{\Gamma}$ intersects of $\operatorname{Aut}\left(\mathfrak{u}_{0}\right)$.

For classical compact simple Lie algebras, both statements of the above proposition fail in general. For example, in $\operatorname{Aut}(\mathfrak{s u}(2 n))$ and for an odd $p$ with $1 \leq p \leq n-1$, $\tau \circ \operatorname{Ad}\left(I_{n, n}\right)(\tau=$ complex conjugation) doesn't commute with any involution conjugate to $\operatorname{Ad}\left(I_{p, 2 n-p}\right)$; in $\operatorname{Aut}(\mathfrak{s o}(4 n))$, Aut $(\mathfrak{s o}(4 n))^{\Gamma_{n}} \subset \operatorname{Int}(\mathfrak{s o}(4 n))$ (see Table 3 for the definition of $\Gamma_{n}$ ).

For each Klein subgroup $\Gamma$ listed in Table 3 or 4 with two generators $\theta, \sigma \in$ $\operatorname{Aut}\left(\mathfrak{u}_{0}\right)$, we get the centralizer $\operatorname{Aut}\left(\mathfrak{u}_{0}\right)^{\Gamma}$ by calculating $\left(\operatorname{Aut}\left(\mathfrak{u}_{0}\right)^{\theta}\right)^{\sigma}$. The results 
about $\operatorname{Aut}\left(\mathfrak{u}_{0}\right)^{\Gamma}$ are listed in Table 5 for classical compact simple Lie algebras and in Table 6 for exceptional compact simple Lie algebras.

\section{References}

[Bahturin and Goze 2008] Y. Bahturin and M. Goze, " $\mathbb{Z}_{2} \times \mathbb{Z}_{2}$-symmetric spaces", Pacific J. Math. 236:1 (2008), 1-21. MR 2009c:17052 Zbl 1156.53030

[Berger 1957] M. Berger, "Les espaces symétriques noncompacts", Ann. Sci. École Norm. Sup. (3) 74 (1957), 85-177. MR 21 \#3516 Zbl 0093.35602

[Bourbaki 2002] N. Bourbaki, Lie groups and Lie algebras, Chapter 4-6, Springer, Berlin, 2002. MR 2003a:17001 Zbl 0983.17001

[Carter 1993] R. W. Carter, Finite groups of Lie type: conjugacy classes and complex characters, Wiley, Chichester, 1993. MR 94k:20020 Zbl 0567.20023

[Chuah and Huang 2010] M.-K. Chuah and J.-S. Huang, "Double Vogan diagrams and semisimple symmetric spaces”, Trans. Amer. Math. Soc. 362:4 (2010), 1721-1750. MR 2011a:17016 Zbl 1205.17008

[Helgason 2001] S. Helgason, Differential geometry, Lie groups, and symmetric spaces, Graduate Studies in Mathematics 34, American Mathematical Society, Providence, RI, 2001. MR 2002b:53081 Zbl 0993.53002

[Helminck 1988] A. G. Helminck, "Algebraic groups with a commuting pair of involutions and semisimple symmetric spaces", Adv. Math. 71:1 (1988), 21-91. MR 90a:17011 Zbl 0685.22007

[Huang 2002] J.-S. Huang, "Admissible square quadruplets and semisimple symmetric spaces", $A d v$. Math. 165:1 (2002), 101-123. MR 2003c:53070 Zbl 1001.22011

[Knapp 2002] A. W. Knapp, Lie groups beyond an introduction, 2nd ed., Progress in Mathematics 140, Birkhäuser, Boston, 2002. MR 2003c:22001 Zbl 1075.22501

[Kollross 2009] A. Kollross, "Exceptional $\mathbb{Z}_{2} \times \mathbb{Z}_{2}$-symmetric spaces", Pacific J. Math. 242:1 (2009), 113-130. MR 2010j:17023 Zbl 1184.53056

[Larsen 1994] M. Larsen, "On the conjugacy of element-conjugate homomorphisms", Israel J. Math. 88:1-3 (1994), 253-277. MR 95k:20073 Zbl 0898.20025

[Lutz 1981] R. Lutz, "Sur la géométrie des espaces $\Gamma$-symétriques”, C. R. Acad. Sci. Paris Sér. I Math. 293:1 (1981), 55-58. MR 82j:53090 Zbl 0474.53047

[Ōshima and Sekiguchi 1984] T. Ōshima and J. Sekiguchi, "The restricted root system of a semisimple symmetric pair", pp. 433-497 in Group representations and systems of differential equations (Tokyo, 1982), edited by K. Okamoto, Adv. Stud. Pure Math. 4, North-Holland, Amsterdam, 1984. MR 87c:17017 Zbl 0577.17004

[Wolf and Gray 1968] J. A. Wolf and A. Gray, "Homogeneous spaces defined by Lie group automorphisms, I”, J. Differential Geometry 2 (1968), 77-114. MR 38 \#4625a Zbl 0169.24103

Received January 17, 2012. Revised July 15, 2012.

JING-SONG HUANG

DEPARTMENT OF MATHEMATICS

Hong Kong University of SCIENCE AND TECHNOLOGY (HKUST)

KOWLOON

HONG KONG SAR

CHINA

mahuang@ust.hk 


\begin{tabular}{|c|c|c|}
\hline $\mathfrak{u}_{0}$ & $\Gamma_{i}$ & $L=\operatorname{Aut}\left(\mathfrak{u}_{0}\right)^{\Gamma_{i}}$ \\
\hline $\mathfrak{s u}(p+q), p \neq q$ & $\Gamma_{p, q}$ & $\left((O(p) \times O(q)) /\left\langle\left(-I_{p},-I_{q}\right)\right\rangle\right) \times\langle\tau\rangle$ \\
\hline $\mathfrak{s u}(2 p)$ & $\Gamma_{p, p}$ & $\begin{array}{l}\left((O(p) \times O(p)) /\left\langle\left(-I_{p},-I_{p}\right)\right\rangle\right) \rtimes\left\langle\tau, J_{p}\right\rangle \\
\quad \operatorname{Ad}\left(J_{p}\right)(X, Y)=(Y, X), \operatorname{Ad}(\tau)=1\end{array}$ \\
\hline $\mathfrak{s u}(2 p)$ & $\Gamma_{p}^{\prime}$ & $\left(U(p) /\left\langle-I_{p}\right\rangle\right) \rtimes\langle\tau, z\rangle, \operatorname{Ad}(z)=1$ \\
\hline $\mathfrak{s u}(2 p+2 q), p \neq q$ & $\Gamma_{p, q}^{\prime}$ & $\left((\operatorname{Sp}(p) \times \operatorname{Sp}(q)) /\left\langle\left(-I_{p},-I_{q}\right)\right\rangle\right) \times\left\langle\tau J_{p+q}\right\rangle$ \\
\hline $\mathfrak{s u}(4 p)$ & $\Gamma_{p, p}^{\prime}$ & $\begin{array}{c}\left((\operatorname{Sp}(p) \times \operatorname{Sp}(p)) /\left\langle\left(-I_{p},-I_{p}\right)\right\rangle\right) \rtimes\left\langle\tau J_{2 p}, J_{p}\right\rangle \\
\quad \operatorname{Ad}\left(J_{p}\right)(X, Y)=(Y, X), \operatorname{Ad}\left(\tau J_{2 p}\right)=1\end{array}$ \\
\hline $\mathfrak{s u}(p+q+r+s)$ & $\Gamma_{p, q, r, s}$ & $\begin{array}{c}\left(\left(S\left(U(p) \times U(q) \times U_{r} \times U_{s}\right) /\left\langle Z_{p+q+r+s}\right\rangle\right) \rtimes\langle\tau\rangle\right. \\
\operatorname{Ad}(\tau)=\text { complex conjugation }\end{array}$ \\
\hline $\mathfrak{s u}(2 p+2 r), p \neq r$ & $\Gamma_{p, p, r, r}$ & $\begin{array}{l}\left(\left(S\left(U(p) \times U(p) \times U_{r} \times U_{r}\right) /\left\langle Z_{2 p+2 r}\right\rangle\right) \rtimes\left\langle\tau, J_{p, r}\right\rangle\right. \\
\quad \operatorname{Ad}\left(J_{p, r}\right)\left(X_{1}, X_{2}, X_{3}, X_{4}\right)=\left(X_{2}, X_{1}, X_{4}, X_{3}\right)\end{array}$ \\
\hline $\mathfrak{s u}(4 p)$ & $\Gamma_{p, p, p, p}$ & $\begin{array}{c}\left(\left(S(U(p) \times U(p) \times U(p) \times U(p)) /\left\langle Z_{4 p}\right\rangle\right) \rtimes\left\langle\tau, J_{2 p}, J_{p, p}\right\rangle\right. \\
\operatorname{Ad}\left(J_{2 p}\right)\left(X_{1}, X_{2}, X_{3}, X_{4}\right)=\left(X_{3}, X_{4}, X_{1}, X_{2}\right)\end{array}$ \\
\hline $\mathfrak{s u}(2 p)$ & $\Gamma_{p}$ & $\begin{array}{c}P S U(p) \rtimes\left\langle F_{p}, \tau\right\rangle \\
\operatorname{Ad}(\tau)=\text { complex conjugation, } \operatorname{Ad}\left(F_{p}\right)=1\end{array}$ \\
\hline $\mathfrak{s o}(p+q+r+s)$ & $\Gamma_{p, q, r, s}$ & $(O(p) \times O(q) \times O(r) \times O(s)) /\left\langle-I_{p+q+r+s}\right\rangle$ \\
\hline $\mathfrak{s o}(2 p+2 r), p \neq r$ & $\Gamma_{p, p, r, r}$ & $\begin{array}{c}\left.\left((O(p) \times O(p) \times O(r) \times O(r)) /\left\langle-I_{2 p+2 r}\right\rangle\right)\right) \rtimes\left\langle J_{p, r}\right\rangle \\
\operatorname{Ad}\left(J_{p, r}\right)\left(X_{1}, X_{2}, X_{3}, X_{4}\right)=\left(X_{2}, X_{1}, X_{4}, X_{3}\right)\end{array}$ \\
\hline $\mathfrak{s o}(4 p), p \neq 2$ & $\Gamma_{p, p, p, p}$ & $\begin{array}{c}\left((O(p))^{4} /\left\langle-I_{4 p}\right\rangle\right) \rtimes\left\langle J_{2 p}, J_{p, p}\right\rangle \\
\operatorname{Ad}\left(J_{2 p}\right)\left(X_{1}, X_{2}, X_{3}, X_{4}\right)=\left(X_{3}, X_{4}, X_{1}, X_{2}\right)\end{array}$ \\
\hline $\mathfrak{s o}(8)$ & $\Gamma_{2,2,2,2}$ & $\begin{array}{c}\left(U(1)^{4} / Z^{\prime}\right) \rtimes\left\langle\epsilon_{1,2}, \epsilon_{1,3}, \epsilon_{1,4}, S_{4}\right\rangle \\
\operatorname{Ad}\left(\epsilon_{1,2}\right)\left(X_{1}, X_{2}, X_{3}, X_{4}\right)=\left(-X_{1},-X_{2}, X_{3}, X_{4}\right), \text { etc } \\
S_{4} \text { acts by permutations }\end{array}$ \\
\hline $\mathfrak{s o}(2 p)$ & $\overline{\Gamma_{p}}$ & $\left(O(p) /\left\langle-I_{p}\right\rangle\right) \times F_{p}$ \\
\hline $\mathfrak{s o}(2 p+2 q), p \neq q$ & $\Gamma_{p, q}$ & $\begin{array}{l}\left((U(p) \times U(q)) /\left\langle\left(-I_{p},-I_{q}\right)\right\rangle\right) \rtimes\langle\tau\rangle \\
\operatorname{Ad}(\tau)=\text { complex conjugation }\end{array}$ \\
\hline $\mathfrak{s o}(4 p)$ & $\Gamma_{p, p}$ & $\begin{array}{c}\left((U(p) \times U(p)) /\left\langle\left(-I_{p},-I_{p}\right)\right\rangle\right) \rtimes\left\langle\tau, J_{p}\right\rangle \\
\operatorname{Ad}\left(J_{p}\right)(X, Y)=(Y, X)\end{array}$ \\
\hline $\mathfrak{s o}(4 p)$ & $\Gamma_{p}^{\prime}$ & $\left(\mathrm{Sp}(p) /\left\langle-I_{p}\right\rangle\right) \times F_{p}^{\prime}$ \\
\hline $\mathfrak{s p}(n)$ & $\Gamma_{p}$ & $\left(O(n) /\left\langle-I_{n}\right\rangle\right) \times F_{p}$ \\
\hline $\mathfrak{s p}(p+q), p \neq q$ & $\Gamma_{p, q}$ & $\left((U(p) \times U(q)) /\left\langle\left(-I_{p},-I_{q}\right)\right\rangle\right) \times\langle\tau\rangle$ \\
\hline $\mathfrak{s p}(2 p)$ & $\Gamma_{p, p}$ & $\begin{array}{c}\left((U(p) \times U(p)) /\left\langle\left(-I_{p},-I_{p}\right)\right\rangle\right) \rtimes\left\langle\tau, J_{p}\right\rangle \\
\operatorname{Ad}(\tau)=\text { complex conjugation, } \operatorname{Ad}\left(J_{p}\right)(X, Y)=(Y, X)\end{array}$ \\
\hline $\mathfrak{s p}(2 p)$ & $\Gamma_{p}^{\prime}$ & $\left(\mathrm{Sp}(p) /\left\langle-I_{p}\right\rangle\right) \times F_{p}^{\prime}$ \\
\hline $\mathfrak{s p}(p+q+r+s)$ & $\Gamma_{p, q, r, s}$ & $(\mathrm{Sp}(p) \times \mathrm{Sp}(q) \times \mathrm{Sp}(r) \times \mathrm{Sp}(s)) /\left\langle-I_{p+q+r+s}\right\rangle$ \\
\hline $\mathfrak{s p}(2 p+2 r), p \neq r$ & $\Gamma_{p, p, r, r}$ & $\begin{array}{c}\left((\mathrm{Sp}(p) \times \mathrm{Sp}(p) \times \mathrm{Sp}(r) \times \mathrm{Sp}(r)) /\left\langle-I_{2 p+2 r}\right\rangle\right) \rtimes\left\langle J_{p, r}\right\rangle \\
\quad \operatorname{Ad}\left(J_{p, r}\right)\left(X_{1}, X_{2}, X_{3}, X_{4}\right)=\left(X_{2}, X_{1}, X_{4}, X_{3}\right)\end{array}$ \\
\hline $\mathfrak{s p}(4 p)$ & $\Gamma_{p, p, p, p}$ & $\begin{array}{c}\left((\operatorname{Sp}(p))^{4} /\left\langle-I_{4 p}\right\rangle\right) \rtimes\left\langle J_{2 p}, J_{p, p}\right\rangle \\
\operatorname{Ad}\left(J_{2 p}\right)\left(X_{1}, X_{2}, X_{3}, X_{4}\right)=\left(X_{3}, X_{4}, X_{1}, X_{2}\right)\end{array}$ \\
\hline
\end{tabular}

Table 5. Fixed point subgroups of Klein four-subgroups: classical cases. 


\begin{tabular}{|c|c|}
\hline $\mathfrak{u}_{0} \quad \Gamma_{i}$ & $L=\operatorname{Aut}\left(\mathfrak{u}_{0}\right)^{\Gamma_{i}}$ \\
\hline $\mathfrak{e}_{6} \quad \Gamma_{1}$ & $\begin{array}{l}\left.U(3) \times S U(3) \times U(1) \times U(1)) /\left\langle\left(e^{\frac{2 \pi i}{3}} I, I, e^{\frac{2 \pi i}{3}}, 1\right),\left(I, e^{\frac{2 \pi i}{3}} I, e^{\frac{-2 \pi i}{3}}, 1\right)\right\rangle\right) \rtimes\langle z, \tau\rangle, \\
\operatorname{Ad}(\tau)(X, Y, \lambda, \mu)=(\bar{Y}, \bar{X}, \lambda, \mu), \operatorname{Ad}(z)(X, Y, \lambda, \mu)=\left(Y, X, \lambda^{-1}, \mu^{-1}\right)\end{array}$ \\
\hline $\mathfrak{e}_{6} \quad \Gamma_{2}$ & $\begin{array}{c}(S U(4) \times \operatorname{Sp}(1) \times \operatorname{Sp}(1) \times U(1)) /\langle(i I,-1,1, i),(I,-1,-1,-1)\rangle) \rtimes\langle\tau\rangle, \\
\operatorname{Ad}(\tau)(X, y, z, \lambda)=\left(J_{2} \bar{X}\left(J_{2}\right)^{-1}, y, z, \lambda^{-1}\right)\end{array}$ \\
\hline $\mathfrak{e}_{6} \quad \Gamma_{3}$ & $(S U(5) \times U(1) \times U(1)) \rtimes\left\langle\tau^{\prime}\right\rangle, \quad \operatorname{Ad}\left(\tau^{\prime}\right)(X, \lambda, \mu)=\left(\bar{X}, \lambda^{-1}, \mu^{-1}\right)$ \\
\hline $\mathfrak{e}_{6} \Gamma_{4}$ & $\begin{array}{c}((\operatorname{Spin}(8) \times U(1) \times U(1)) /\langle(-1,-1,1),(c, 1,-1)\rangle) \rtimes\langle\tau\rangle \\
\operatorname{Ad}(\tau)(x, \lambda, \mu)=\left(x, \lambda^{-1}, \mu^{-1}\right)\end{array}$ \\
\hline $\mathfrak{e}_{6} \quad \Gamma_{5}$ & $((\operatorname{Sp}(3) \times \operatorname{Sp}(1)) /\langle(-I,-1)\rangle) \times\langle\tau\rangle$ \\
\hline $\mathfrak{e}_{6} \quad \Gamma_{6}$ & $\begin{array}{c}((S O(6) \times U(1)) /\langle(-I,-1)\rangle) \rtimes\left\langle\tau^{\prime}, z\right\rangle \\
\operatorname{Ad}(z)(X, \lambda)=\left(I_{3,3} X I_{3,3}, \lambda^{-1}\right), \operatorname{Ad}\left(\tau^{\prime}\right)=1\end{array}$ \\
\hline $\begin{array}{ll}\mathfrak{e}_{6} & \Gamma_{7}\end{array}$ & $\operatorname{Spin}(9) \times\langle\tau\rangle$ \\
\hline $\mathfrak{e}_{6} \quad \Gamma_{8}$ & $((\operatorname{Spin}(5) \times \operatorname{Spin}(5)) /\langle(-1,-1)\rangle) \rtimes\left\langle\tau^{\prime}, z\right\rangle, \quad \operatorname{Ad}(z)(x, y)=(y, x)$ \\
\hline $\mathfrak{e}_{7} \Gamma_{1}$ & $\begin{array}{c}\left((S U(6) \times U(1) \times U(1)) /\left\langle\left(e^{\frac{2 \pi i}{3}} I, e^{\frac{-2 \pi i}{3}}, 1\right),(-I, 1,1)\right\rangle\right) \rtimes\langle z\rangle \\
\operatorname{Ad}(z)(X, \lambda, \mu)=\left(J_{3} \bar{X} J_{3}^{-1}, \lambda^{-1}, \mu^{-1}\right)\end{array}$ \\
\hline $\mathfrak{e}_{7} \quad \Gamma_{2}$ & $\left(\operatorname{Spin}(8) \times \operatorname{Sp}(1)^{3}\right) /\langle(c,-1,1,1),(1,-1,-1,-1),(-1,-1,-1,1)\rangle$ \\
\hline $\mathfrak{e}_{7} \Gamma_{3}$ & $\begin{array}{l}((\operatorname{Spin}(10) \times U(1) \times U(1)) /\langle(c, i, 1)\rangle) \rtimes\langle z\rangle \\
\operatorname{Ad}(z)(x, \lambda, \mu)=\left(e_{1} x e_{1}^{-1}, \lambda^{-1}, \mu^{-1}\right)\end{array}$ \\
\hline $\mathfrak{e}_{7} \Gamma_{4}$ & $\begin{array}{c}\left((S U(6) \times \operatorname{Sp}(1) \times U(1)) /\left\langle\left(e^{\frac{2 \pi i}{3}} I, 1, e^{\frac{-2 \pi i}{3}}\right),(-I,-1,1)\right\rangle\right) \rtimes\langle z\rangle \\
\operatorname{Ad}(z)(X, y, \lambda)=\left(J_{3} \bar{X} J_{3}^{-1}, y, \lambda^{-1}\right)\end{array}$ \\
\hline $\begin{array}{ll}\mathfrak{e}_{7} & \Gamma_{5}\end{array}$ & $\begin{array}{c}\left((\operatorname{Spin}(6) \times \operatorname{Spin}(6) \times U(1)) /\left\langle\left(c, c^{\prime}, 1\right),(1,-1,-1)\right\rangle\right) \rtimes\left\langle z_{1}, z_{2}\right\rangle, \\
\operatorname{Ad}\left(z_{1}\right)(x, y, \lambda)=\left(y, x, \lambda^{-1}\right), \operatorname{Ad}\left(z_{2}\right)(x, y, \lambda)=\left(e_{1} x e_{1}^{-1}, e_{1} y e_{1}^{-1}, \lambda^{-1}\right)\end{array}$ \\
\hline $\mathfrak{e}_{7} \quad \Gamma_{6}$ & $F_{4} \times\langle\tau, \omega\rangle$ \\
\hline $\mathfrak{e}_{7} \Gamma_{7}$ & $(\mathrm{Sp}(4) /\langle-I\rangle) \times\left\langle\tau, \omega^{\prime}\right\rangle$ \\
\hline $\mathfrak{e}_{7} \Gamma_{8}$ & $(S O(8) /\langle-I\rangle) \times\left\langle\tau^{\prime}, \omega^{\prime}\right\rangle$ \\
\hline $\mathfrak{e}_{8} \Gamma_{1}$ & $\left(\left(E_{6} \times U(1) \times U(1)\right) /\left\langle\left(c, e^{\frac{2 \pi i}{3}}, 1\right)\right\rangle\right) \rtimes\langle z\rangle, \quad \mathfrak{l}_{0}^{z}=\mathfrak{f}_{4} \oplus 0 \oplus 0$ \\
\hline $\mathfrak{e}_{8} \quad \Gamma_{2}$ & $(\operatorname{Spin}(12) \times \operatorname{Sp}(1) \times \operatorname{Sp}(1)) /\langle(c,-1,1),(-1,-1,-1)\rangle$ \\
\hline $\mathfrak{e}_{8} \quad \Gamma_{3}$ & $((S U(8) \times U(1)) /\langle(-I, 1),(i I,-1)\rangle) \rtimes\langle z\rangle, \quad \mathfrak{l}_{0}^{z}=\mathfrak{s p}(4) \oplus 0$ \\
\hline $\mathfrak{e}_{8} \quad \Gamma_{4}$ & $((\operatorname{Spin}(8) \times \operatorname{Spin}(8)) /\langle(-1,-1),(c, c)\rangle) \rtimes\langle z\rangle, \quad \operatorname{Ad}(z)(x, y)=(y, x)$ \\
\hline $\mathfrak{f}_{4} \quad \Gamma_{1}$ & $\left((S U(3) \times U(1) \times U(1)) /\left\langle\left(e^{\frac{2 \pi i}{3}} I, e^{\frac{-2 \pi i}{3}}, 1\right)\right\rangle\right) \rtimes\langle z\rangle, \quad \mathfrak{l}_{0}^{z}=\mathfrak{s o}(3) \oplus 0 \oplus 0$ \\
\hline $\mathfrak{f}_{4} \quad \Gamma_{2}$ & $((\mathrm{Sp}(2) \times \mathrm{Sp}(1) \times \mathrm{Sp}(1)) /\langle(-I,-1,-1)\rangle$ \\
\hline $\mathfrak{f}_{4} \quad \Gamma_{3}$ & $\operatorname{Spin}(8)$ \\
\hline $\mathfrak{g}_{2} \quad \Gamma$ & $(U(1) \times U(1)) \rtimes\langle z\rangle, \quad \operatorname{Ad}(z)(\lambda, \mu)=\left(\lambda^{-1}, \mu^{-1}\right)$ \\
\hline
\end{tabular}

Table 6. Fixed point subgroups of Klein four-subgroups: exceptional cases.

JUN YU

DEPARTMENT OF MATHEMATICS

ETH ZURICH

8092 ZURICH

SWITZERLAND

jun.yu@math.ethz.ch 


\title{
PACIFIC JOURNAL OF MATHEMATICS
}

\author{
msp.org/pjm
}

Founded in 1951 by E. F. Beckenbach (1906-1982) and F. Wolf (1904-1989)

\section{EDITORS}

V. S. Varadarajan (Managing Editor)

Department of Mathematics

University of California

Los Angeles, CA 90095-1555

pacific@math.ucla.edu

Paul Balmer

Department of Mathematics

University of California

Los Angeles, CA 90095-1555

balmer@math.ucla.edu

Daryl Cooper

Department of Mathematics

University of California

Santa Barbara, CA 93106-3080 cooper@math.ucsb.edu

Jiang-Hua $\mathrm{Lu}$

Department of Mathematics

The University of Hong Kong

Pokfulam Rd., Hong Kong jhlu@maths.hku.hk
Don Blasius

Department of Mathematics University of California

Los Angeles, CA 90095-1555

blasius@math.ucla.edu

Robert Finn

Department of Mathematics Stanford University

Stanford, CA 94305-2125

finn@math.stanford.edu

Sorin Popa

Department of Mathematics

University of California

Los Angeles, CA 90095-1555

popa@math.ucla.edu

Paul Yang

Department of Mathematics

Princeton University

Princeton NJ 08544-1000

yang@math.princeton.edu

\section{PRODUCTION}

Silvio Levy, Scientific Editor, production@msp.org

\section{SUPPORTING INSTITUTIONS}

ACADEMIA SINICA, TAIPEI

CALIFORNIA INST. OF TECHNOLOGY

INST. DE MATEMÁTICA PURA E APLICADA

KEIO UNIVERSITY

MATH. SCIENCES RESEARCH INSTITUTE

NEW MEXICO STATE UNIV.

OREGON STATE UNIV.

\author{
STANFORD UNIVERSITY \\ UNIV. OF BRITISH COLUMBIA \\ UNIV. OF CALIFORNIA, BERKELEY \\ UNIV. OF CALIFORNIA, DAVIS \\ UNIV. OF CALIFORNIA, LOS ANGELES \\ UNIV. OF CALIFORNIA, RIVERSIDE \\ UNIV. OF CALIFORNIA, SAN DIEGO \\ UNIV. OF CALIF., SANTA BARBARA
}

\author{
Vyjayanthi Chari \\ Department of Mathematics \\ University of California \\ Riverside, CA 92521-0135 \\ chari@math.ucr.edu \\ Kefeng Liu \\ Department of Mathematics \\ University of California \\ Los Angeles, CA 90095-1555 \\ liu@math.ucla.edu \\ Jie Qing \\ Department of Mathematics \\ University of California \\ Santa Cruz, CA 95064 \\ qing@cats.ucsc.edu
}

These supporting institutions contribute to the cost of publication of this Journal, but they are not owners or publishers and have no responsibility for its contents or policies.

See inside back cover or msp.org/pjm for submission instructions.

The subscription price for 2013 is US \$400/year for the electronic version, and \$485/year for print and electronic.

Subscriptions, requests for back issues and changes of subscribers address should be sent to Pacific Journal of Mathematics, P.O. Box 4163, Berkeley, CA 94704-0163, U.S.A. The Pacific Journal of Mathematics is indexed by Mathematical Reviews, Zentralblatt MATH, PASCAL CNRS Index, Referativnyi Zhurnal, Current Mathematical Publications and the Science Citation Index.

The Pacific Journal of Mathematics (ISSN 0030-8730) at the University of California, c/o Department of Mathematics, 798 Evans Hall \#3840, Berkeley, CA 94720-3840, is published monthly except July and August. Periodical rate postage paid at Berkeley, CA 94704, and additional mailing offices. POSTMASTER: send address changes to Pacific Journal of Mathematics, P.O. Box 4163, Berkeley, CA 94704-0163.

PJM peer review and production are managed by EditFLOW ${ }^{\circledR}$ from Mathematical Sciences Publishers.

PUBLISHED BY

mathematical sciences publishers

nonprofit scientific publishing

http://msp.org/

(C) 2013 Mathematical Sciences Publishers 


\section{PACIFIC JOURNAL OF MATHEMATICS}

Volume $262 \quad$ No. $2 \quad$ April 2013

Certifying incompressibility of noninjective surfaces with scl

DANNY CALEGARI

Global well-posedness for the 3D rotating Navier-Stokes equations with highly oscillating initial data

QIONGLEI CHEN, CHANGXING MiAO and ZHIFEI ZHANG

Presenting Schur superalgebras

HOUSSEIN El TURKEY and JONATHAN R. KUJAWA

Classifying zeros of two-sided quaternionic polynomials and computing zeros of two-sided polynomials with complex coefficients

FENG LIANGGUI and ZHAO KAIMING

Coxeter groups, imaginary cones and dominance

XIANG FU

Semicontinuity of automorphism groups of strongly pseudoconvex domains: The low differentiability case

Robert E. Greene, Kang-Tae Kim, Steven G. Krantz and

AERYEONG SEO

Klein four-subgroups of Lie algebra automorphisms

JING-SONG HUANG and JUN YU

Fractal entropy of nonautonomous systems

Rui KuANG, WEN-ChiaO CHENG and BING LI

A GJMS construction for 2-tensors and the second variation of the total

$Q$-curvature

YoshiniKo MaTSUMOTO

Droplet condensation and isoperimetric towers

Matteo Novaga, Andrei Sobolevski and Eugene Stepanov

Brauer's height zero conjecture for metacyclic defect groups

BENJAMIN SAMBALE 